\title{
Non-linear seismic analysis of RC structures with energy-dissipating devices
}

\author{
P. Mata ${ }^{1, *,}$, A. H. Barbat ${ }^{1}$, S. Oller ${ }^{1}$ and R. Boroschek ${ }^{2}$ \\ ${ }^{1}$ Technical University of Catalonia, UPC, Jordi Girona 1-3, Mòdul C1, Campus Nord, 08034 Barcelona, Spain \\ ${ }^{2}$ Civil Engineering Department, University of Chile, Blanco Encalada 2002, Santiago, Chile
}

\begin{abstract}
SUMMARY
The poor performance of some reinforced concrete (RC) structures during strong earthquakes has alerted about the need of improving their seismic behavior, especially when they are designed according to obsolete codes and show low structural damping, important second-order effects and low ductility, among other defects. These characteristics allow proposing the use of energy-dissipating devices for improving their seismic behavior. In this work, the non-linear dynamic response of RC buildings with energy dissipators is studied using advanced computational techniques. A fully geometric and constitutive non-linear model for the description of the dynamic behavior of framed structures is developed. The model is based on the geometrically exact formulation for beams in finite deformation. Points on the cross section are composed of several simple materials. The mixing theory is used to treat the resulting composite. A specific type of element is proposed for modeling the dissipators including the corresponding constitutive relations. Special attention is paid to the development of local and global damage indices for describing the performance of the buildings. Finally, numerical tests are presented for validating the ability of the model for reproducing the non-linear seismic response of buildings with dissipators. Copyright (C) 2008 John Wiley \& Sons, Ltd.
\end{abstract}

Received 8 February 2008; Revised 7 October 2008; Accepted 28 October 2008

KEY WORDS: RC structures; beam model; non-linear analysis; composites; passive control

\section{INTRODUCTION}

Conventional seismic design practice permits designing reinforced concrete $(\mathrm{RC})$ structures for forces lower than those expected from the elastic response, on the premise that the structural design

\footnotetext{
*Correspondence to: P. Mata, Technical University of Catalonia, Jordi Girona 1-3, Mòdul C1, Campus Nord, 08034 Barcelona, Spain.

${ }^{\dagger}$ E-mail: pmata@cimne.upc.edu

Contract/grant sponsor: European Commission; contract/grant number: CEE-FP6 Project FP6-50544(GOCE) Contract/grant sponsor: Spanish Government (Ministerio de Educación y Ciencia); contract/grant numbers: BIA200308700-C03-02, BIA2005-06952

Contract/grant sponsor: CIMNE and AIRBUS; contract/grant number: PBSO-13-06
} 
assures significant structural ductility [1]. Frequently, the dissipative zones are located near the beam-column joints and, due to cyclic inelastic incursions during earthquakes, structural elements can suffer a great amount of damage [2]. This situation is generally considered economically acceptable if life safety and collapse prevention are achieved.

In the last decades, new techniques based on adding devices to the buildings with the main objective of dissipating the energy exerted by the earthquake and alleviating the ductility demand on primary structural elements have contributed to improve the seismic behavior of the structures $[3,4]$. Their purpose is to control the seismic response of the buildings by means of a set of dissipating devices that constitutes the control system, adequately located in the structure. In the case of passive energy-dissipating devices (EDD), an important part of the energy input is absorbed and dissipated, therefore, concentrating the non-linear phenomenon in the devices without the need of an external energy supply.

Several works showing the ability of EDDs in controlling the seismic response of structures are available; for example, in Reference [5] the responses of framed structures equipped with viscoelastic and viscous devices are compared; in Reference [6] a comparative study considering metallic and viscous devices is carried out. Aiken [7] presents the contribution of extra energy dissipation due to EDDs as an equivalent damping added to the linear bare structure (the term bare is used to indicate the structure without EDDs or any type of stiffeners) and gives displacement reduction factors as a function of the added damping ratio. A critical review of reduction factors and design force leveis can be consutted in Reference [8]. A mothod for the preliminary design

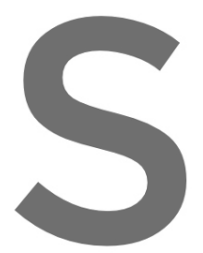
of passively controll accuracy of estimatin they are replaced by and design of structu Today, only a few cour d buildin
heir ener
cos with
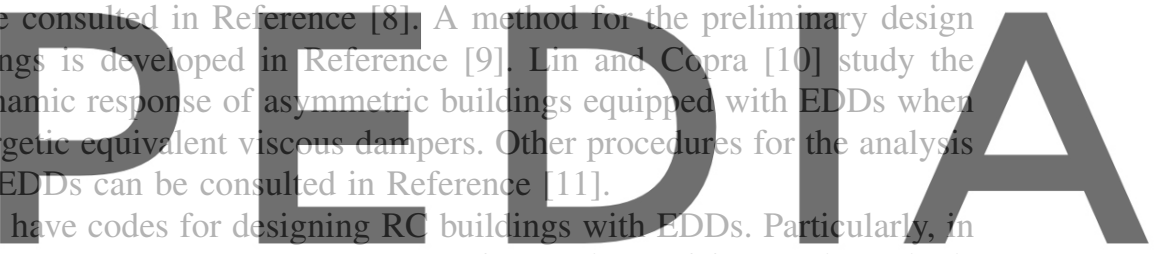

United States the US Federal Emergency Management Agency gives code provisions and standards

The design methods proposed for RC structures are mainly based on the assumption that the behavior of the bare structure remains elastic, while the energy dissipation relies on the control system. However, experimental and theoretical evidence shows that inelastic behavior can also occur in the structural elements of controlled building during severe earthquakes [14]. In order to perform a precise dynamic non-linear analysis of passively controlled buildings, sophisticated numerical tools become necessary for both academics and practitioners [15].

There is agreement that fully three-dimensional numerical technique constitute the most precise tools for the simulation of the seismic behavior of RC buildings. However, the computing time usually required for real structures makes many applications unpractical. Considering that most of the elements in RC buildings are columns and beams, one-dimensional formulations for structural elements appear as a solution combining both numerical precision and reasonable computational costs [16]. Experimental evidence shows that inelasticity in beam elements can be formulated in terms of cross-sectional quantities [17]. Some formulations of this type have been extended for considering geometric non-linearities $[18,19]$. An additional refinement is obtained considering inhomogeneous distributions of materials on arbitrarily shaped beam cross sections [20]. In this case, the constitutive relationship at the cross-sectional level is deduced by integration and, therefore, the mechanical behavior of beams with complex combinations of materials can be simulated. 
On the one hand, formulations for beams considering both constitutive and geometric nonlinearity are rather scarce; most of the geometrically non-linear models are limited to the elastic case [21,22] and the inelastic behavior has been mainly restricted to plasticity [18]. Recently, Mata et al. $[16,23]$ have extended the geometrically exact formulation for beams due to Reissner and Simo $[22,24,25]$ to an arbitrary distribution of composite materials on the cross sections for the static and dynamic cases.

On the other hand, from the numerical point of view, EDDs usually have been described in a global sense by means of force-displacement or moment-curvature relationships [4], which attempt to capture appropriately the energy-dissipating capacity of the devices [26]. The inclusion of EDDs in software packages for the seismic analysis of RC structures is frequently done by linking elements equipped with the mentioned non-linear relationships. The relative displacement and/or rotation between the anchorage points activates the dissipative mechanisms of the devices.

In summary, a modern numerical approach to the structural seismic analysis of RC buildings should take into account the following aspects:

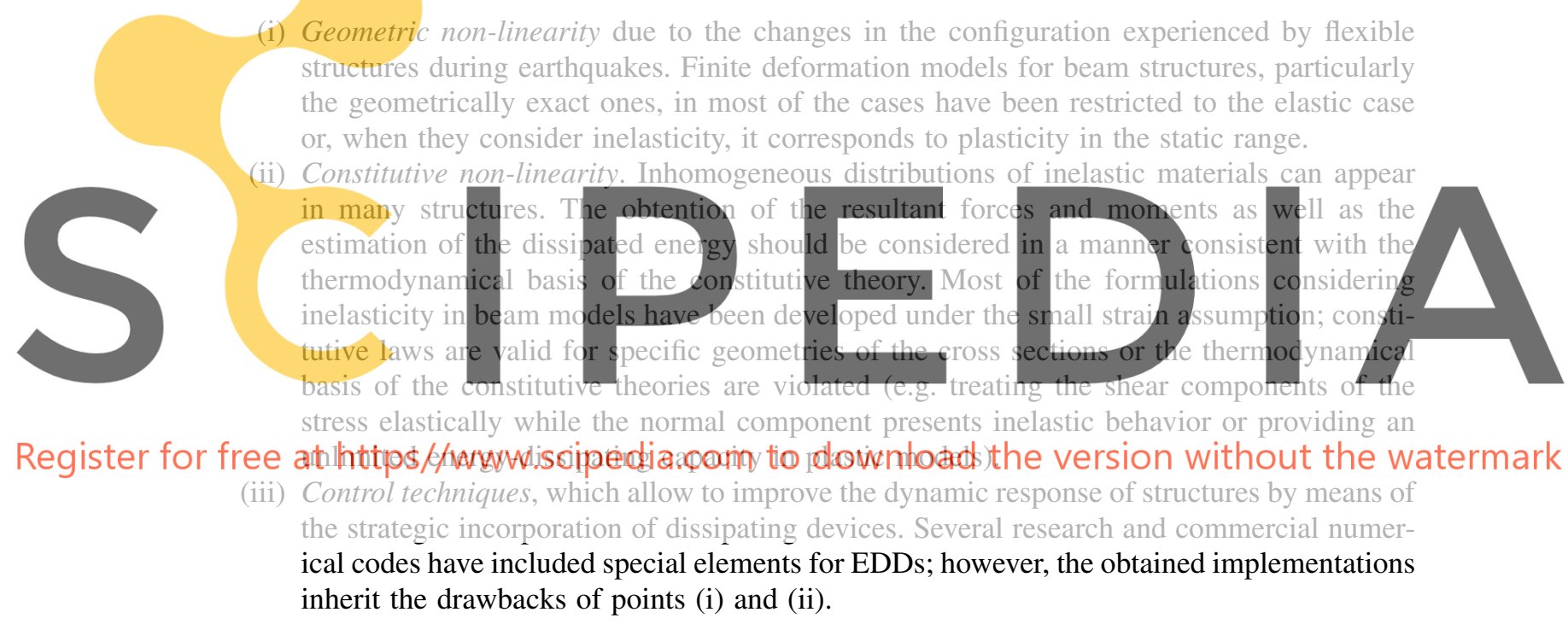

According to the authors's knowledge the state of the art in seismic analysis provides a set of partial solutions to the above-mentioned requirements; however, there is not a unified approach covering all these aspects in a manner consistent with the principles of the continuum mechanics (see (ii)).

In this work, a fully geometric and constitutive non-linear formulation for rod elements is extended to the case of flexible RC structures equipped with EDDs. A fiber-like approach is used to represent arbitrary distributions of composite materials on the beam cross sections. EDDs are considered as bar elements linking two points in the structure. Thermodynamically consistent constitutive laws are used for concrete and longitudinal and transversal steel reinforcements. In particular, a damage model able to treat the degradation associated with the tensile and compressive components of stress in an independent manner is presented. The extension to the rate-dependent case is obtained by means of a regularization of the evolution rules of the damage thresholds. The mixing rule is employed for the treatment of the resulting composite. A specific non-linear hysteretic force-displacement relationship is provided for describing the mechanical behavior of 
several types of EDDs. A description of the damage indices capable of estimating the remaining load-carrying capacity of the buildings is also given. Finally, numerical results from simulations showing the ability of the proposed formulation in simulating the static and dynamic inelastic response of RC buildings equipped with EDDs are provided. Examples cover several complex phenomena such as the inelastic $P-\Delta$ effect and inelastic dynamic structural torsion.

\section{FINITE DEFORMATION FORMULATION FOR STRUCTURAL ELEMENTS}

\subsection{Beam model}

The original geometrically exact formulation for beams due to Reissner [24], Simo [22] and Simo and Vu Quoc $[25,27]$ is expanded for considering an intermediate curved reference configuration according to Ibrahimbegović [21]. The main difficulty arises from the fact that the geometry and the kinematics of the beams are developed in the non-linear differential manifold $\mathbb{R}^{3} \times \mathrm{SO}(3)$ and, therefore, a number of standard procedures, such as the computation of strain measures or the linearization of the weak form of the balance equations become more complicated. In this section a brief summary of results relevant for the development of constitutive laws able to be incorporated in the beam theory and the construction of a model for EDDs are presented.
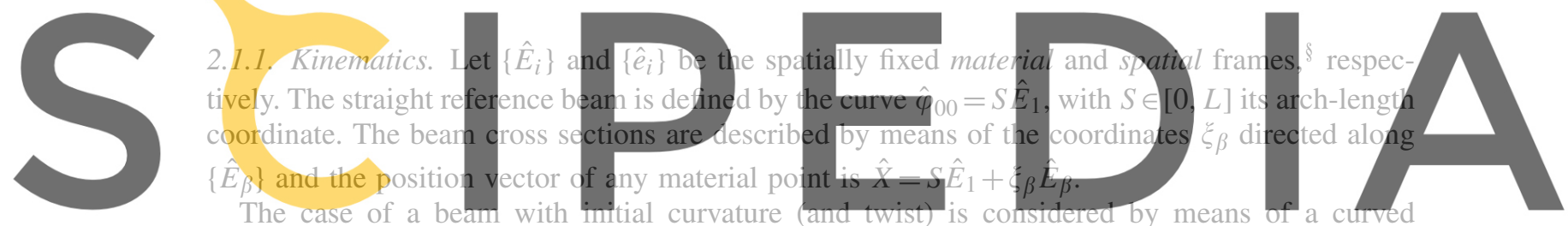

reference beam defined by means of the spatially fixed curve $\hat{\varphi}_{0}=\varphi_{0 i}(S) \hat{e}_{i} \in \mathbb{R}^{3}$. Each point on

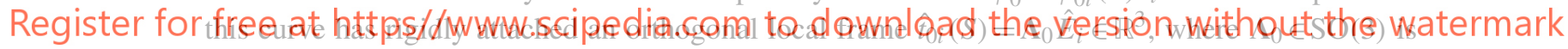

the orientation tensor. The beam cross section $\mathscr{A}(S)$ is defined considering the local coordinate system $\xi_{\beta}$ but directed along $\left\{\hat{t}_{0 \beta}\right\}$. The planes of the cross sections are normal to the vector tangent to the reference curve, i.e. $\hat{\varphi}_{0, S}=\hat{t}_{01}(S)$. The position vector of a material point on the curved reference beam is $\hat{x}_{0}=\hat{\varphi}_{0}+\boldsymbol{\Lambda}_{0} \xi_{\beta} \hat{E}_{\beta}$. In this case, the straight beam is used as an auxiliary reference frame for the construction of strain measures as it will be explained in following.

The motion displaces points on the curved reference beam from $\hat{\varphi}_{0}(S)$ to $\hat{\varphi}(S, t)$ (at time $t$ ) adding a translational displacement $\hat{u}(S, t)$ and the local orientation frame is simultaneously rotated, together with the beam cross section, from $\Lambda_{0}(S)$ to $\Lambda(S, t)$ by means of the incremental rotation tensor $\boldsymbol{\Lambda}=\boldsymbol{\Lambda}_{n} \boldsymbol{\Lambda}_{0} \equiv \hat{t}_{i} \otimes \hat{E}_{i} \in \mathrm{SO}$ (3) (see Figure 1).

In general, the normal vector $\hat{t}_{1} \neq \hat{\varphi}_{, S}$ because of the shearing [22]. The position vector of a material point on the current beam is

$$
\hat{x}\left(S, \xi_{\beta}, t\right)=\hat{\varphi}(S, t)+\xi_{\beta} \hat{t}_{\beta}(S, t)=\hat{\varphi}+\boldsymbol{\Lambda} \xi_{\beta} \hat{E}_{\beta}
$$

\footnotetext{
¥The symbol $\mathrm{SO}(3)$ is used to denote the finite rotation manifold $[22,27]$.

${ }^{\S}$ The indices $i$ and $\beta$ range over $\{1,2,3\}$ and $\{2,3\}$, respectively, and summation convention hold. The symbol $(\cdot)_{, x}$ is used to denote partial differentiation of $(\cdot)$ with respect to $x$.
} 


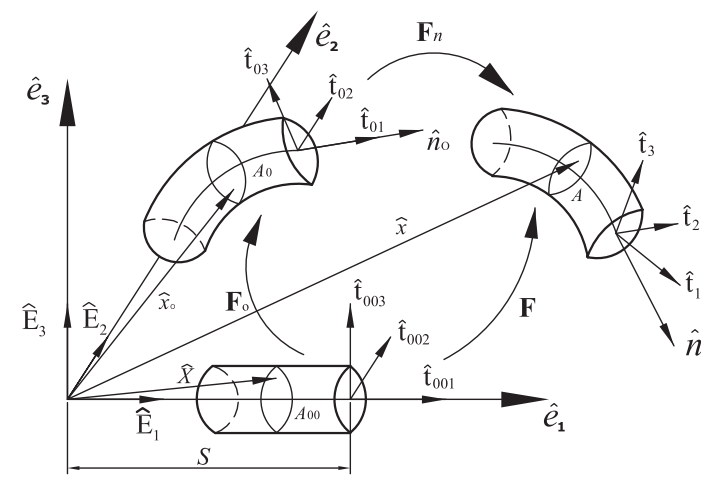

Figure 1. Configurational description of the beam.

Equation (1) implies that the current beam configuration is completely determined by the pairs $(\hat{\varphi}, \Lambda) \in \mathbb{R}^{3} \times \operatorname{SO}(3)$.
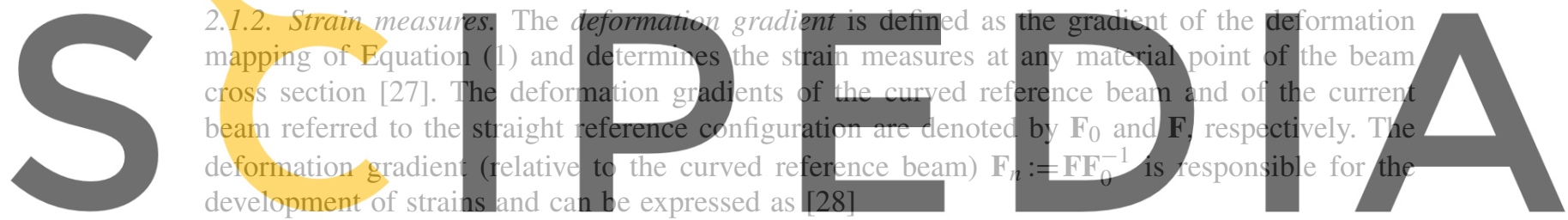

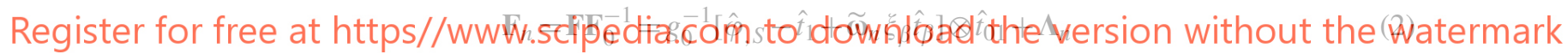

where $g_{0}=\operatorname{Det}\left[\mathbb{F}_{0}\right]$ and $\widetilde{\omega}_{n} \equiv \Lambda_{n, S} \Lambda_{n}^{\mathrm{T}}$ is the spatial curvature tensor relative to the curved reference beam. In Equation (2), the term defined as $\hat{\gamma}_{n}=\hat{\varphi}_{S}-\hat{t}_{1}$ corresponds to the reduced spatial strain measure of shearing and elongation [22,28] with material description given by $\hat{\Gamma}=\boldsymbol{\Lambda}^{\mathrm{T}} \hat{\gamma}$. The material representation of $\mathbf{F}_{n}$ is obtained as $\mathbf{F}_{n}^{\mathrm{m}}=\boldsymbol{\Lambda}^{\mathrm{T}} \mathbf{F}_{n} \boldsymbol{\Lambda}_{0}$.

Removing the rigid body component from $\mathbf{F}_{n}$, it is possible to construct the spatial strain tensor $\boldsymbol{\varepsilon}_{n}=\mathbf{F}_{n}-\boldsymbol{\Lambda}_{n}$. The corresponding spatial strain vector acting on the current beam cross section is obtained as

$$
\hat{\varepsilon}_{n}=\boldsymbol{\varepsilon}_{n} \hat{t}_{01}=g_{0}^{-1}\left[\hat{\gamma}_{n}+\widetilde{\boldsymbol{\omega}}_{n} \xi_{\beta} \hat{t}_{\beta}\right]
$$

with material form given by $\hat{\mathscr{E}}_{n}=\boldsymbol{\Lambda}^{\mathrm{T}} \hat{\varepsilon}_{n}$.

2.1.3. Stress measures. $\varepsilon_{n}$ is (energetically) conjugated to the asymmetric First Piola Kirchhoff (FPK) stress tensor $\mathbf{P}=\hat{P}_{i} \otimes \hat{t}_{0 i}$ [22] with $\hat{P}_{i}$ being the FPK stress vector acting on the deformed face in the current beam corresponding to the normal $\hat{t}_{0 i}$ in the curved reference configuration. Equivalently, $\hat{\varepsilon}_{n}$ is the energetically conjugated pair to $\hat{P}_{1}$. The corresponding material forms are 
given by and $\hat{P}_{1}^{\mathrm{m}}=\boldsymbol{\Lambda}^{\mathrm{T}} \hat{P}_{1}$, respectively. Additionally, the spatial form of the stress resultant $\hat{n}$ and the stress couple $\hat{m}$ vectors can be computed according to

$$
\hat{n}(S)=\int_{\mathscr{A}} \hat{P}_{1} \mathrm{~d} \mathscr{A}, \quad \hat{m}(S)=\int_{\mathscr{A}}(\hat{x}-\hat{\varphi}) \times \hat{P}_{1} \mathrm{~d} \mathscr{A}
$$

The material forms of $\mathbf{P}, \hat{n}$ and $\hat{m}$ are obtained by means of the pullback operation as $\mathbf{P}^{\mathrm{m}}=\boldsymbol{\Lambda}^{\mathrm{T}} \mathbf{P} \boldsymbol{\Lambda}_{0}$, $\hat{n}^{\mathrm{m}}=\boldsymbol{\Lambda}^{\mathrm{T}} \hat{n}$ and $\hat{m}^{\mathrm{m}}=\boldsymbol{\Lambda}^{\mathrm{T}} \hat{m}$, respectively.

\section{Remark 1}

It is worth noting that due to the assumption made in Equation (1), the following restrictions appear on the components of the strain and stress tensors: (i) $\mathscr{E}_{n}=\boldsymbol{\Lambda}^{\mathrm{T}} \boldsymbol{\varepsilon}_{n} \boldsymbol{\Lambda}=\hat{\mathscr{E}}_{n} \otimes \hat{E}_{1}, \quad\left(\boldsymbol{\varepsilon}_{n} \hat{t}_{\beta}=0\right)$ and, therefore, (ii) $\mathrm{P}^{\mathrm{m}}=\hat{P}_{1}^{\mathrm{m}} \otimes \hat{E}_{1}$.

\subsubsection{Strain rate measures. An objective measure of the strain rate vector $\hat{s}_{n}$ acting on any material point on the current beam cross section can be deduced using the results presented in [23] and using the definition of the Lie derivative operator $[\ulcorner]$ given in [16], as follows:}
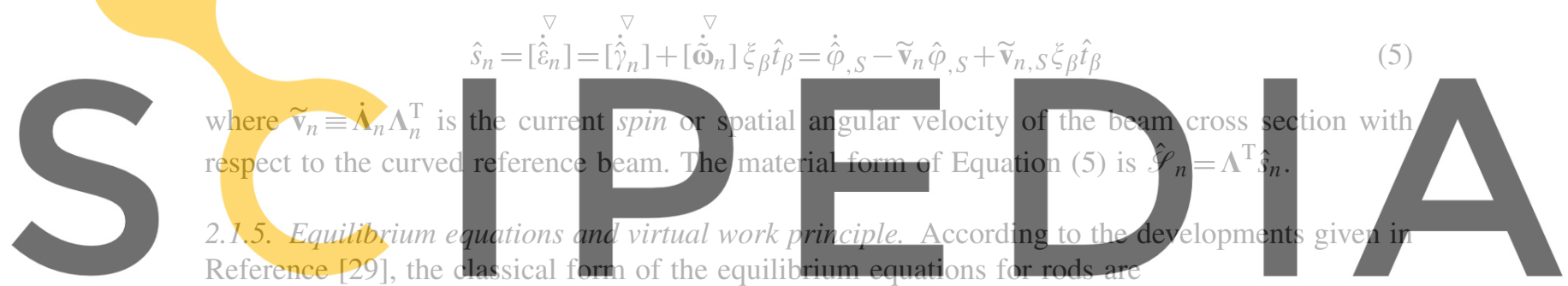

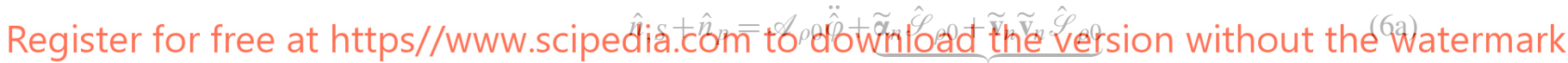

$$
\hat{m}_{, S}+\hat{\varphi}_{, S} \times \hat{n}+\hat{m}_{p}=\mathscr{I}_{\rho 0} \hat{\alpha}_{n}+\widetilde{\mathbf{v}}_{n} \mathscr{I}_{\rho 0} \hat{v}_{n}+\underbrace{\hat{\mathscr{S}}_{\rho 0} \times \ddot{\hat{\varphi}}}_{D_{2}}
$$

where $\hat{n}_{p}$ and $\hat{m}_{p}$ are the external body force and body moment per unit of reference length at time $t, \mathscr{A}_{\rho 0}, \hat{\mathscr{S}}_{\rho 0}$ and $\mathscr{I}_{\rho 0}$ are the cross-sectional mass density, the first mass moment density and the second mass moment density per unit of length of the curved reference beam, respectively; their explicit expressions can be consulted in References [27, 28]. $\widetilde{\boldsymbol{\alpha}}_{n} \equiv \ddot{\boldsymbol{\Lambda}}_{n} \boldsymbol{\Lambda}_{n}^{\mathrm{T}}-\widetilde{\mathbf{v}}_{n} \widetilde{\mathbf{v}}_{n}$ is the angular acceleration of the beam cross section and $\hat{v}_{n}$ and $\hat{\alpha}_{n}$ are the axial vectors of $\widetilde{\mathbf{v}}_{n}$ and $\widetilde{\alpha}_{n}$, respectively. For most of the practical cases, the terms $D_{1}$ and $D_{2}$ can be neglected or added to the external forces and moments.

Considering a kinematically admissible variation ${ }^{\mathbb{I}} \hat{h} \equiv(\delta \hat{\varphi}, \delta \hat{\theta})$ of the pair $(\hat{\varphi}, \boldsymbol{\Lambda})$ [27], taking the dot product with Equations (6a) and (6b), integrating over the length of the curved reference beam and integrating by parts, we obtain the following non-linear functional $G_{w}(\hat{\varphi}, \boldsymbol{\Lambda}, \hat{h})$ corresponding

\footnotetext{
"Supposing that $\Lambda$ is parameterized in terms of the spatial rotation vector and following the results of References $[21,22]$ it is possible to show that $\delta \boldsymbol{\Lambda}=\delta \tilde{\boldsymbol{\theta}} \boldsymbol{\Lambda}$ with $\delta \hat{\theta}=\operatorname{axial}[\delta \tilde{\boldsymbol{\theta}}] \in \mathbb{R}^{3}$ an admissible infinitesimal rotation.
} 
to the weak form of the balance equations [21,27], which is another way of writing the virtual work principle:

$$
\begin{aligned}
G_{w}(\hat{\varphi}, \boldsymbol{\Lambda}, \hat{h})= & \int_{0}^{L}\left[\left(\delta \hat{\varphi}_{, S}-\delta \hat{\theta} \times \hat{\varphi}_{, S}\right) \cdot \hat{n}+\delta \hat{\theta}_{, S} \cdot \hat{m}\right] \mathrm{d} S \\
& +\int_{0}^{L}\left[\delta \hat{\varphi} \cdot \mathscr{A}_{\rho_{0}} \ddot{\hat{\varphi}}+\delta \hat{\theta} \cdot\left(\mathscr{I}_{\rho_{0}} \hat{\alpha}_{n}+\hat{\mathbf{v}}_{n} \mathscr{I}_{\rho_{0}} \hat{v}_{n}\right)\right] \mathrm{d} S \\
& -\left(\int_{0}^{L}\left[\delta \hat{\varphi} \cdot \hat{n}_{p}+\delta \hat{\theta} \cdot \hat{m}_{p}\right] \mathrm{d} S+\left.[\delta \hat{\varphi} \cdot \hat{n}+\delta \hat{\theta} \cdot \hat{m}]\right|_{0} ^{L}\right) \\
= & G_{w}^{\operatorname{int}}(\hat{\varphi}, \Lambda, \hat{h})+G_{w}^{\text {ine }}(\hat{\varphi}, \Lambda, \hat{h})-G_{w}^{\operatorname{ext}}(\hat{\varphi}, \Lambda, \hat{\Phi}, \hat{h})=0
\end{aligned}
$$

where $\hat{\Phi}=\left[\hat{n}_{p}^{\mathrm{T}}, \hat{m}_{p}^{\mathrm{T}}\right]$ is the external loading vector, $G_{w}^{\mathrm{int}}$, and $G_{w}^{\mathrm{ine}}$ and $G_{w}^{\text {ext }}$ correspond to the internal, inertial and external contributions of the virtual work principle. The terms $\left(\delta \hat{\varphi}_{\text {. }}-\delta \hat{\theta} \times \hat{\varphi}_{\text {. }}\right)$ and $\delta \hat{\theta}_{, S}$ appearing in Equation (7) correspond to the co-rotated variations of the reduced strain measures $\hat{\gamma}_{n}$ and $\hat{\omega}_{n}$ in spatial description.

\subsection{Energy-dissipating devices}

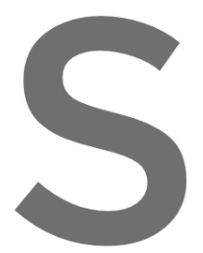

The finite deformation model fo releasing the rotational of the device is descri middle of the resulting degrees current position of a p bar. I
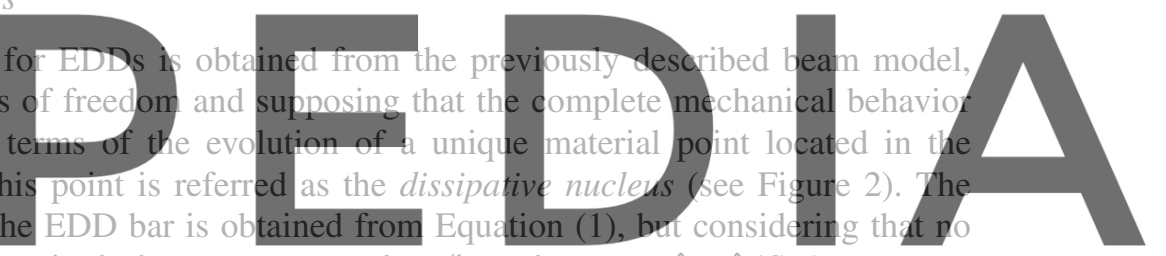
cross-sectional description is required; thus, one can neglect $\xi_{\beta}$ and assume $\hat{x}=\hat{\varphi}(S, t)$.

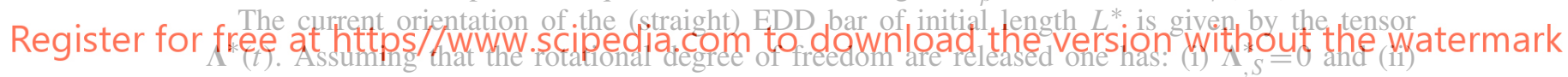

$\dot{\Lambda}^{*} \neq 0$. Therefore, the spatial position of the dissipative nuclei is obtained as $\hat{\varphi}\left(L^{*} / 2, t\right)$ where $L^{*} / 2$ is the arch-length coordinate of the middle point in the bar element.

The only non-zero component of the strain vector is the axial one, denoted by $\mathscr{E}_{d_{1}}$ and computed with the help of Equation (3) as

$$
\mathscr{E}_{d_{1}}(t)=\left.\hat{\Gamma}_{n}\right|_{\left(L^{*} / 2\right)} \cdot \hat{E}_{1}=\left.\left[\left(\boldsymbol{\Lambda}^{* \mathrm{~T}} \hat{\varphi}_{, S}\right) \cdot \hat{E}_{1}\right]\right|_{\left(L^{*} / 2\right)}-1=\left.\left[\hat{\varphi}_{, S} \cdot \hat{t}_{1}\right]\right|_{\left(L^{*} / 2\right)}-1
$$

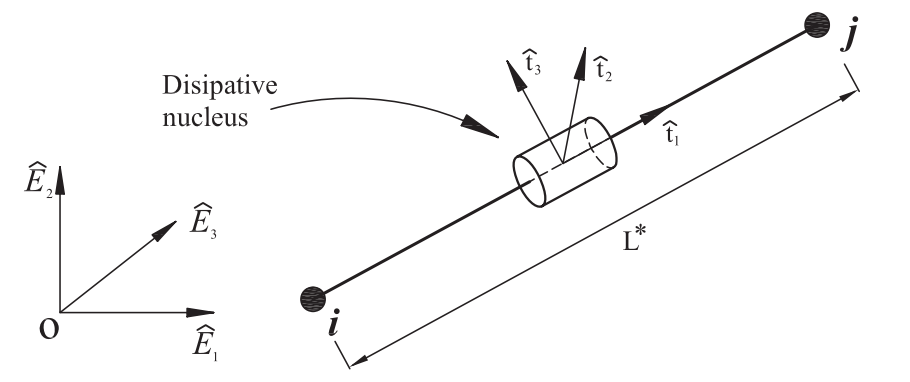

Figure 2. Energy-dissipating device. 
and the corresponding strain rate, $\dot{\mathscr{E}}_{d_{1}}$, is obtained as

$$
\dot{\mathscr{E}}_{d_{1}}(t)=\left.\frac{\mathrm{d}}{\mathrm{d} t} \mathscr{E}_{d_{1}}(t)\right|_{\left(L^{*} / 2, t\right)}=\left.\left[\left(\boldsymbol{\Lambda}^{* \mathrm{~T}}\left(\dot{\hat{\varphi}}_{, S}-\widetilde{\mathbf{v}}_{n} \hat{\varphi}_{, S}\right)\right) \cdot \hat{E}_{1}\right]\right|_{\left(L^{*} / 2, t\right)}
$$

Note that the same expression can be deduced from Equation (5). Let $P_{d}^{\mathrm{m}}$ be the value of the stress measure in the EDD, which is energetically conjugated to $\mathscr{E}_{d_{1}}$. The formulation of specific constitutive relations $P_{d}^{\mathrm{m}}\left(\mathscr{E}_{d_{1}}, \dot{\mathscr{E}}_{d_{1}}\right)$ is provided in Section 3.5 .

In spite of the fact that $\hat{t}_{1}$ can be calculated as $\hat{t}_{1}=\left(\hat{\varphi}\left(L^{*}\right)-\hat{\varphi}(0)\right) /\left\|\hat{\varphi}\left(L^{*}\right)-\hat{\varphi}(0)\right\|=\boldsymbol{\Lambda} \hat{E}_{1}$ one has that $\delta \hat{t}_{1}=\delta \boldsymbol{\Lambda} \hat{E}_{1}=\delta \widetilde{\boldsymbol{\theta}} \boldsymbol{\Lambda} \neq 0$ although $\delta \hat{\varphi}$ can be zero for rigid body motions (however, $\left.(\delta \hat{\theta})_{, S}=0\right)$. Then, taking $(\delta \hat{\varphi}, \delta \hat{\theta})$ superposed onto $(\hat{\varphi}, \boldsymbol{\Lambda})$ and considering that the linear part of Equation (8) is $\delta \mathscr{E} d_{1}=\left[\delta \hat{\varphi}_{, S}+\widetilde{\varphi}, S \delta \hat{\theta}\right] \cdot \hat{t}_{1}$, one has that the contribution of the EDDs to the functional of Equation (7) written in the material description is

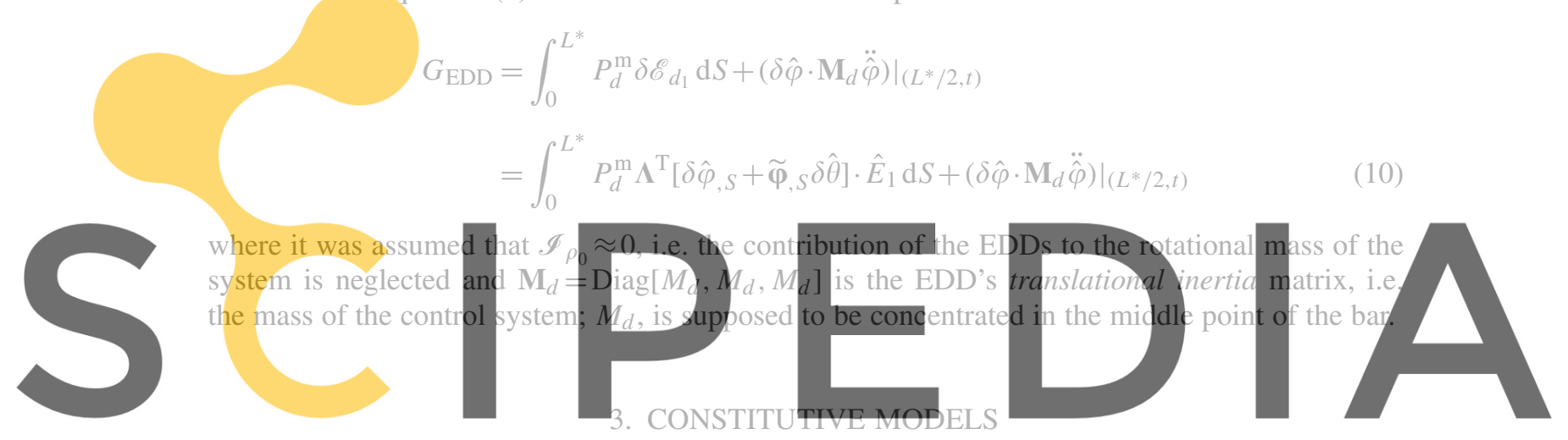

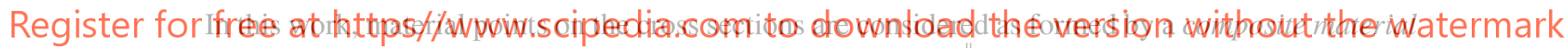
corresponding to a homogeneous mixture of different simplell components, each of them with its own constitutive law (see Figure 3). The resulting behavior is obtained by means of the mixing theory (see e.g. [30] or more recently [31,32] and references therein).

In the formulation of constitutive models, the kinematic assumptions of the present theory has to be considered, which limit the number of known components of the strain and stress tensors to those existing on the cross-sectional planes. Therefore, following the same reasonings as in previous works of the authors [16,23], the models are formulated in terms of the material form of the FPK stress, strain and strain rate vectors, $\hat{P}_{1}^{\mathrm{m}}, \hat{\mathscr{E}}_{n}$ and $\hat{S}_{n}$, respectively.

Although the present constitutive models constitute a dimensionally reduced form of the general three-dimensional formulations, they allow to simulate the coupled non-linear behavior among the components of the stress vector, respecting the thermodynamical basis of irreversible processes. In this sense, the present approach avoids the use of one-dimensional constitutive laws for the axial component of the stress maintaining the behavior of shear components uncoupled, which is one of the most common assumptions in fiber-like models for rods (see e.g. [33, 34]). Two kinds of non-linear constitutive models for simple materials are used: the tension-compression damage and the plasticity models.

\footnotetext{
"The term simple is used for referring to materials that are described by means of a single constitutive law.
} 


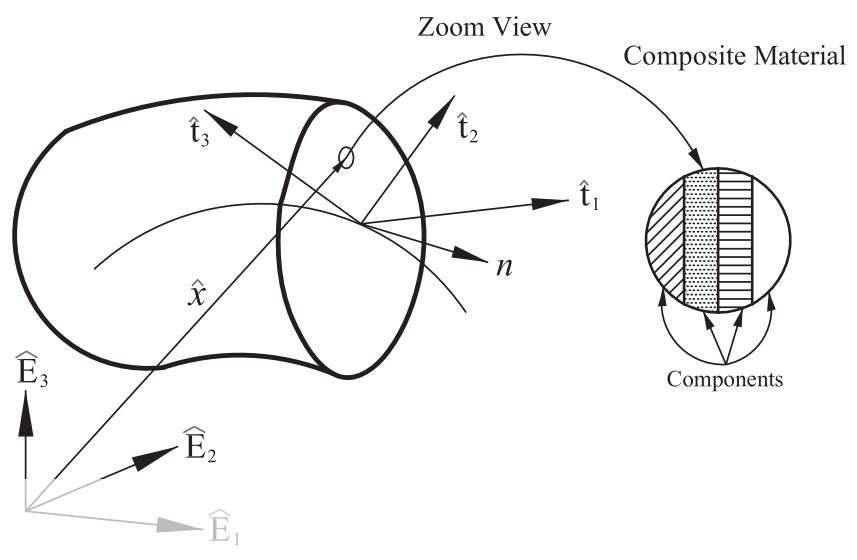

Figure 3. Cross section showing the composite associated with a material point.

\subsection{Degrading materials: tension-compression damage model}

Continuum damage mechanics provides a general framework for the derivation of thermodynamically consistent mode firstly introduced by however, representati [38] or, more recen variable model that empirical relations
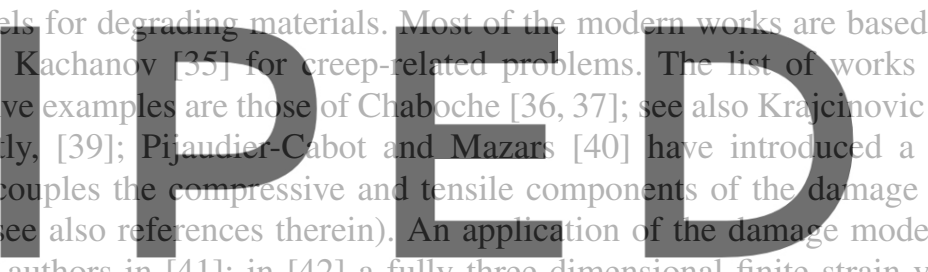

is provided by same authors in [41]; in [42] a fully three-dimensional finite strain visco-damage

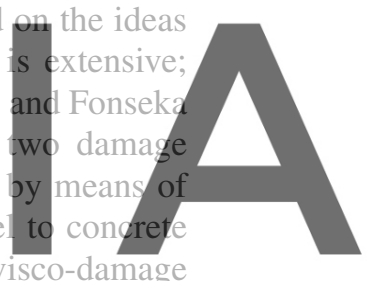

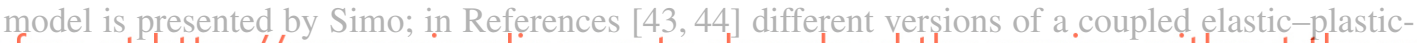

finite deformation can be consulted in $[45,46]$, respectively.

In this work the tension-compression damage model presented by Faria et al. [47] is modified in order to allow its inclusion in the Reissner-Simo formulation for inelastic rods. These modifications permit to consider two important features of the mechanical behavior of concrete:

(i) Independent degradation of the mechanical properties for tensile or compressive loading paths that allows to simulate the crack-closure observed during cyclical loading (stiffness recovery).

(ii) Large differences in the tensile and compressive thresholds (see Figure 4).

The model is based on an adequate form of the free energy density depending on two (independent) scalar damage variables** $d^{ \pm} \in[0,1]$, related to the degradation mechanisms occurring under tensile $(+)$ or compressive $(-)$ stress concentrations.

In summary, the formulation of the model is based on splitting the material form of the FPK stress tensor, written only in terms of its cross-sectional components, into its tensile and compressive parts. A suitable form of the Helmholtz-free energy density [48], along with the Clausius-Duheim

\footnotetext{
**In the following, the superscript \pm is used to denote a quantity defined for both, the tensile case, related to $(+)$ and the compressive case, related to $(-)$.
} 


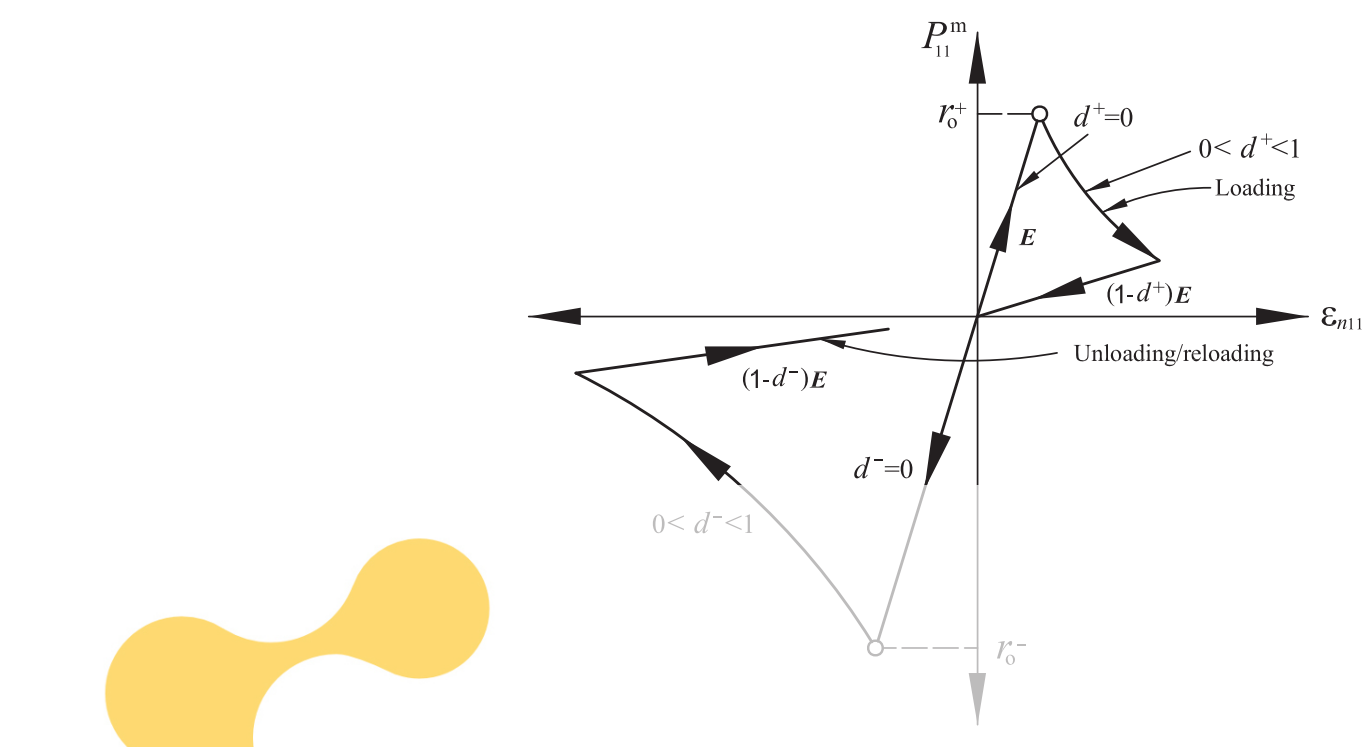

Figure 4. Tension-compression damage model.

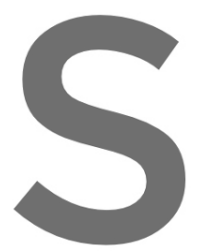

inequality and the Coleman's method, is used f
[49]. Then, the evolution equations for the in
and tangent relation are established. Finally, a

3.1.1. Stress split. Let $\overline{\mathbf{P}}^{\mathrm{m}}=\hat{\bar{P}}_{1}^{\mathrm{m}} \otimes \hat{E}_{1}=\left[\mathscr{C}^{\mathrm{me}} \hat{\mathscr{E}}_{n}\right] \otimes \hat{E}_{1}$ be the material form of the elastic FPK

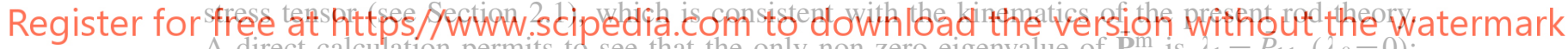
A direct calculation permits to see that the only non-zero eigenvalue of $\mathbb{P}^{\mathrm{m}}$ is $\lambda_{1}=P_{11}\left(\lambda_{\beta}=0\right)$ :

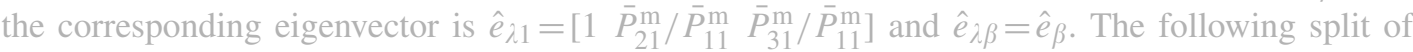
the stress tensor is proposed:

$$
\begin{aligned}
& \overline{\mathbf{P}}^{\mathrm{m}+}=\left\langle\lambda_{1}\right\rangle \hat{e}_{\lambda 1} \otimes \hat{e}_{\lambda 1}=\hat{\bar{P}}_{i}^{\mathrm{m}+} \otimes \hat{E}_{i} \\
& \overline{\mathbf{P}}^{\mathrm{m}-}=\overline{\mathbf{P}}^{\mathrm{m}}-\overline{\mathbf{P}}^{\mathrm{m}+}=\hat{\bar{P}}_{i}^{\mathrm{m}-} \otimes \hat{E}_{i}
\end{aligned}
$$

Then, the Helmholtz-free energy potential of the degrading model [48] is given by

$$
\Psi^{ \pm}\left(\hat{\mathscr{E}}_{n}, d^{ \pm}\right)=\left(1-d^{ \pm}\right) \Psi_{0}^{ \pm}=\frac{1}{2}\left[\left(1-d^{+}\right) \overline{\mathbf{P}}^{\mathrm{m}+}: \mathbf{C}_{0}^{-1}: \overline{\mathbf{P}}^{\mathrm{m}}+\left(1-d^{-}\right) \overline{\mathbf{P}}^{\mathrm{m}-}: \mathbf{C}_{0}^{-1}: \overline{\mathbf{P}}^{\mathrm{m}}\right]
$$

where $\mathbf{C}_{0 i j k l}^{-1}=\alpha_{1}\left(\delta_{i k} \delta_{j l}+\delta_{i l} \delta_{j k}\right)+\alpha_{2} \delta_{i j} \delta_{k l}$ is the fourth-order elastic compliance tensor with $\alpha_{1}=(1+v) / 2 E$ and $\alpha_{2}=-v / E$. Note that if $d^{ \pm}=0, \Psi^{ \pm}$corresponds to the elastic stored energy. The kinematic assumptions of Section 2.1 imply that $\mathbf{C}_{0}^{-1}: \overline{\mathbf{P}}^{\mathrm{m}}=\mathscr{E}_{n}=g^{-1} \hat{\mathscr{E}}_{n} \otimes \hat{E}_{1}$ and, after some algebraic manipulations, Equation (12) can be rewritten as

$$
\Psi^{ \pm}=\frac{1}{2}\left(1-d^{ \pm}\right)\left[\left(2 \alpha_{1}+\alpha_{2}\right) \bar{P}_{11}^{\mathrm{m}} \bar{P}_{11}^{\mathrm{m} \pm}+\alpha_{1} \bar{P}_{\beta 1}^{\mathrm{m}} \bar{P}_{\beta 1}^{\mathrm{m} \pm}\right]
$$


In this manner, the free energy density is expressed only in terms of the cross-sectional stress vector and two situations can occur:

(i) Tensile axial behavior, $\lambda_{1}=\bar{P}_{11}^{\mathrm{m}}>0$, then $\overline{\mathbf{P}}^{\mathrm{m}+} \neq 0$ (see Equation (11a)) and

$$
\begin{aligned}
\hat{\bar{P}}_{i}^{\mathrm{m}+} & =\frac{\bar{P}_{i 1}^{\mathrm{m}}}{\bar{P}_{11}^{\mathrm{m}}} \hat{\bar{P}}_{1}^{\mathrm{m}}, \quad \hat{\bar{P}}_{1}^{\mathrm{m}-}=0 \\
\Psi^{+} & =\frac{1}{2}\left(1-d^{+}\right)\left[\frac{1-v}{2 E} \lambda_{1}^{2}+\frac{1+v}{2 E}\left\|\hat{P}_{1}^{\mathrm{m}}\right\|^{2}\right]=\frac{1}{2}\left(1-d^{+}\right) \Psi_{0}^{+}
\end{aligned}
$$

Considering $0 \leqslant d^{ \pm} \leqslant 1$, it is possible to see that $\Psi^{+}>0$. This case applies for zones in the cross section that are subjected to tension due to flexion or only tensile axial force.

(ii) Compressive axial behavior, $\lambda_{1}=\bar{P}_{11}^{\mathrm{m}}<0$, then $\overline{\mathbf{P}}^{\mathrm{m}+}=0, \overline{\mathbf{P}}^{\mathrm{m}-}=\hat{\bar{P}}_{1}^{\mathrm{m}} \otimes \hat{E}_{1}$ (see Equation (11b)) and $\Psi^{-}=\frac{1}{2}\left(1-d^{-}\right) \Psi_{0}^{-}>0$. This case considers compressed zones in flexion or compressive axial force. The case $\lambda_{1} \approx 0$ reduces to $\Psi^{-}=\frac{1}{2}\left(1-d^{-}\right) \alpha_{1}\left\|\hat{P}_{1}^{\mathrm{m}}\right\|^{2}>0$.

It is worth to note that $-\partial \Psi / \partial d^{ \pm}=\Psi_{0}^{ \pm}$are the thermodynamical forces associated with $d^{ \pm}$.

\section{Remark 2}

Owing to the kinematic assumptions, the evolution of shear stresses depends on the sign of $\lambda_{1}$ and not on the their own sign. An additional sophistication can be obtained considering, for example, the stress tensor $\overline{\mathbf{P}}_{*}^{\mathrm{m}}=\frac{1}{2}\left[\overline{\mathbf{P}}^{\mathrm{m}}+\overline{\mathbf{P}}^{\mathrm{m} T}\right]$; however, in this case $\left\{\lambda_{\beta}\right\} \neq 0$ and, therefore, more complicated expressions are obtained for Equations (11a)-(13).

3.1.2. Damage criteria. Two (scalar) equivalent stresses, which constitute norms for different stress states [50], are defined as

$$
\begin{aligned}
& \bar{\tau}^{+}:=\sqrt{\overline{\mathbf{P}}^{\mathrm{m}+}: \mathbf{C}_{0}^{-1}: \overline{\mathbf{P}}^{\mathrm{m}+}}= \begin{cases}\sqrt{\Psi_{0}^{+}} & \text {if } \lambda_{1}>0 \\
0 & \text { if } \lambda_{1} \leqslant 0\end{cases} \\
& \bar{\tau}^{-}:=\sqrt{\sqrt{3}\left(K \bar{\sigma}_{\mathrm{oct}}^{-}+\bar{\tau}_{\mathrm{oct}}^{-}\right)}= \begin{cases}0 & \text { if } \lambda_{1}>0 \\
\approx K \bar{P}_{11}^{\mathrm{m}} & \text { if } \lambda_{1} \leqslant 0\end{cases}
\end{aligned}
$$

where $\bar{\sigma}_{\text {oct }}^{-}=\frac{1}{3} \bar{P}_{11}^{\mathrm{m}}, \bar{\tau}_{\text {oct }}^{-}=\frac{\sqrt{2}}{3} \bar{P}_{11}^{\mathrm{m}}$ are the octahedral normal and shear stresses obtained from $\overline{\mathbf{P}}^{\mathrm{m}-}$ and $K \in[1.16-1.2]$ is a materials property, described in detail in Reference [47]. It is worth noting that Equation (15a) is a measure of elastic stored energy in contrast with Equation (15b) where equivalent stress is a measure involving information about specific components of the stress in the space.

In the same way, two separated damage criteria $[51,52]$ are defined

$$
g^{ \pm}\left(\bar{\tau}^{ \pm}, r^{ \pm}\right)=\bar{\tau}^{ \pm}-r^{ \pm} \leqslant 0
$$

where $r^{ \pm}$are the current damage thresholds that control the size of the damage surface. In the present damage theory, the linear-elastic domain depends on $\left\|\hat{P}_{1}^{\mathrm{m}}\right\|^{2}$ and, due to the kinematic assumptions, multi-dimensional representations in the $\lambda_{i}$ planes are not possible. 
The initial values for $r^{ \pm}$are

$$
r_{0}^{+}=\frac{f_{0}^{+}}{\sqrt{E}}, \quad r_{0}^{-}=K f_{0}^{-}
$$

where $f_{0}^{ \pm}$are the elastic thresholds in one-dimensional tensile and compressive tests, respectively. Then, from Equation (15b) it is possible to see that the boundary of the compressive elastic domain is defined for a value of $\bar{r}^{-} K$-times greater than the one-dimensional compressive elastic limit $f_{0}^{-}$.

3.1.3. Evolution equations. The following evolution laws are used for $d^{ \pm}$and $r^{ \pm}$

$$
\dot{d}^{ \pm}=\dot{\theta}^{ \pm} \frac{\partial G^{ \pm}\left(r^{ \pm}\right)}{\partial r^{ \pm}}, \quad \dot{r}^{ \pm}=\dot{\theta}^{ \pm}
$$

where $G^{ \pm}$are monotonically increasing functions determined according with experimental data and $\dot{\theta}^{ \pm}$are the damage consistency parameters. The above rules have to be complemented with the loading and unloading relations defined with the help of the standard Kuhn-Tucker relations:

$$
\text { (i) } \dot{\theta}^{ \pm} \geqslant 0, \quad \text { (ii) } G^{ \pm}>0, \quad \text { (iii) } \dot{\theta}^{ \pm} G^{ \pm}=0
$$

The corresponding interpretation of these relations is standard and can be consulted, e.g. in References [47,53].

In a generic instant $t$ one has that $r_{t}^{ \pm}=\max \left[r_{0}^{ \pm}, r_{*}^{ \pm}\right]$with $r_{*}^{ \pm}=\max _{s \in[0, t]} r_{\mathrm{s}}^{ \pm}$. Finally, from Equations (18) one obtains that $\dot{d}^{ \pm}=\dot{G}^{ \pm}\left(r^{ \pm}\right) \geqslant 0$.

3.1.4. Constitutive relation and dissipation. Considering Equation (12) for the free energy density and the fact that $\mathbf{C}_{0}^{-1}: \mathscr{E}_{n}=\hat{\mathscr{E}}_{1} \otimes \hat{E}_{1}$ one has that Clausius-Duheim inequality [48]: $\dot{\Phi}=-\dot{\Psi}+\hat{P}_{1}^{\mathrm{m}}$. $\dot{\hat{\mathscr{E}}}_{n} \geqslant 0$ can be expressed as

$$
\dot{\Phi}=\left(\hat{P}_{1}^{\mathrm{m}}-\frac{\partial \Psi}{\partial \hat{\mathscr{E}}_{n}}\right) \cdot \dot{\hat{\mathscr{E}}}_{n}+\Psi_{0}^{ \pm} \dot{d}^{ \pm}
$$

which establish that entropy always grows leading to an irreversible process. Considering that both $\overline{\mathbf{P}}^{ \pm}$are first degree homogeneous functions of $\mathscr{E}_{n}$ (see [47] for details) and applying Coleman's principle, the following constitutive relation is obtained:

$$
\hat{P}_{1}^{\mathrm{m}}=\left(1-d^{ \pm}\right) \frac{\partial \Psi_{0}^{ \pm}}{\partial \hat{\mathscr{E}}_{n}}=\left(1-d^{+}\right) \hat{\bar{P}}_{1}^{\mathrm{m}+}+\left(1-d^{-}\right) \hat{\bar{P}}_{1}^{\mathrm{m}-}
$$

where $\hat{\bar{P}}_{1}^{\mathrm{m} \pm}=\overline{\mathbf{P}}^{\mathrm{m}} \hat{E}_{1}$. Taking into account Equation (20) it is straightforward to see that dissipation is given by $\dot{\Phi}=\Psi_{0}^{ \pm} \dot{d}^{ \pm} \geqslant 0$.

The material form of the tangent stiffness tensor, $\mathscr{C}^{\mathrm{mt}}$, is obtained taking the material time derivative of Equation (21) as

$$
\dot{\hat{P}}_{1}^{\mathrm{m}}=\left(1-d^{ \pm}\right) \dot{\overline{\hat{P}}}_{1}^{\mathrm{m} \pm}-\dot{d}^{ \pm} \hat{\bar{P}}_{1}^{\mathrm{m} \pm}=\mathscr{C}^{\mathrm{mt}} \hat{S}_{n}
$$

where $\hat{S}_{n}$ is the material form of the strain rate vector as explained in Section 2.1. 
On the one hand, the time derivatives of $\hat{\bar{P}}_{1}^{\mathrm{m} \pm}$ are obtained considering the kinematic restrictions and results presented in [54] as

$$
\dot{\overline{\vec{P}}}_{1}^{\mathrm{m} \pm}=\mathbf{U}^{ \pm} \mathscr{C}^{\mathrm{me}} \hat{S}_{n}, \quad \mathbf{U}^{+}=\mathscr{H}\left(\lambda_{1}\right) \hat{e}_{\lambda_{1}} \otimes \hat{e}_{\lambda_{1}}, \quad \mathbf{U}^{-}=\mathbf{I}-\mathbf{U}^{+}
$$

where $\mathscr{H}(x)=x+|x| / 2|x|$ is the Heaviside function. Note that due to the fact that the present form of the tension-compression damage theory is restricted to the information existing in the material points belonging to the cross-sectional planes, one has that $\mathbf{U}^{ \pm}=\overline{\mathbf{P}}^{ \pm} / \lambda_{1}$.

On the other hand, the time derivatives of $d^{ \pm}$are computed considering Equations (15a), (15b), (18) and the consistency condition $\dot{r}^{ \pm}=\dot{\bar{\tau}}^{ \pm}$as

$$
\begin{aligned}
& \dot{d}^{ \pm}=\frac{\partial G^{ \pm}}{\partial r^{ \pm}} \dot{\bar{\tau}}^{ \pm}=G^{ \pm \prime} \dot{\bar{\tau}}^{ \pm} \\
& \dot{r}^{+}=\frac{1}{\sqrt{\Psi_{0}^{+}}} \dot{\Psi}_{0}^{+}=\frac{\left[(1-v) \downarrow \hat{\bar{P}}_{1}^{\mathrm{m}}+(1+v) \hat{\bar{P}}_{1}^{\mathrm{m}}\right]}{\sqrt{\Psi_{0}^{+}} E} \cdot \hat{\mathscr{S}}_{n}=\hat{\mathbb{L}}^{+} \cdot \hat{\mathscr{S}}_{n} \\
& \dot{r}^{-}=K \dot{\bar{P}}_{11}^{\mathrm{m}}=K \mathscr{C}^{\mathrm{me}} \hat{E}_{1} \cdot \hat{\mathscr{S}}_{n}=\hat{\mathbb{L}}^{-} \cdot \hat{\mathscr{S}}_{n}
\end{aligned}
$$

where $\mathbb{J}_{i j}=1$ only if $i=j=1$ and 0 otherwise. Finally, replacing Equations (23)-(24c) into (23), using the fact that $\left(\hat{v}_{1} \cdot \hat{v}_{2}\right) \hat{v}_{3}=\left(\hat{v}_{3} \otimes \hat{v}_{1}\right) \hat{v}_{2}$ and after rearranging terms, one obtains

$$
\mathscr{C}^{\mathrm{mt}}=\left[\left(1-d^{ \pm}\right) \mathbf{U}^{ \pm} \mathscr{C}^{\mathrm{me}}-G^{ \pm \prime} \hat{\bar{P}}_{1}^{\mathrm{m}} \otimes \hat{\mathbb{L}}^{ \pm}\right] \quad \text { if } \dot{r}^{ \pm}>0
$$

If unloading has place $\mathscr{C}^{\mathrm{mt}}=\left[\left(1-d^{ \pm}\right) \mathbf{U}^{ \pm} \mathscr{C}^{\mathrm{me}}\right]$. Note that, in general, $\mathscr{C}^{\mathrm{mt}}$ is a un-symmetric stress-dependent tensor.

3.1.5. Integration algorithm. The numerical integration of the constitutive model is carried out in a strain driven fashion as it is usual in displacement-based finite element approaches.

The integration of the damage variables is obtained providing suitable expressions for $d^{ \pm}=$ $G^{ \pm}\left(r^{ \pm}\right)$subjected to the conditions

$$
0 \leqslant G^{ \pm}\left(r^{ \pm}\right) \leqslant 1, \quad \dot{G}^{ \pm}\left(r^{ \pm}\right) \geqslant 0, \quad G^{ \pm}\left(r_{0}^{ \pm}\right)=0
$$

where the first condition enforces the values of the internal variables $d^{ \pm}$to remain in $[0,1]$; the second one ensures that $G^{ \pm}$are monotonically increasing functions as defined in Equation (18) and the third one provides an initial zero damage for the intact material.

In this work, the following expression is used:

$$
d^{ \pm}\left(r^{ \pm}\right)=G^{ \pm}\left(r^{ \pm}\right)=1-\frac{e^{A^{ \pm}\left(1-r^{ \pm}\right)}}{r^{ \pm}}, \quad A^{ \pm}=\left[\frac{E G_{\mathrm{f}}^{ \pm}}{l_{\mathrm{c}} f_{0}^{ \pm}}-\frac{1}{2}\right]^{-1}
$$

which corresponds to materials presenting softening ${ }^{\dagger \dagger}$ immediately after the yielding threshold has been overcome [56]. In Equation (27), the parameter $A^{ \pm}$is calibrated according to the specific

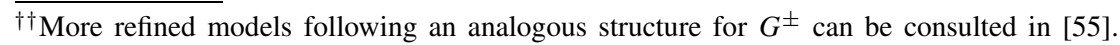


Table I. Flow chart for the damage model.

1. INPUT: material form of $\hat{\mathscr{E}}_{n}$ at times $t_{n}, t_{n+1}$ and iteration $i$, existing on a given integration point on the beam cross section, then

$$
\Delta\left[\hat{\mathscr{E}}_{n}\right]_{n}^{i}=\left[\hat{\mathscr{E}}_{n}\right]_{n+1}^{i}-\left[\hat{\mathscr{E}}_{n}\right]_{n}
$$

2. Compute the material form of the trial (iterative) elastic FPK stress vector as

$$
\left[\hat{\bar{P}}_{1}^{\mathrm{m}}\right]_{n+1}^{\operatorname{trial}(i)}=\left[\hat{\bar{P}}_{1}^{\mathrm{m}}\right]_{n}+\mathscr{C}^{\mathrm{me}} \Delta\left[\hat{\mathscr{E}}_{n}\right]_{n}^{i}
$$

3. From $\left[\hat{\bar{P}}_{1}^{\mathrm{m}}\right]_{n+1}^{\text {trial }(i)}$ determine $\lambda_{1}$ and $\hat{e}_{\lambda_{1}}$. Then construct the split of $\overline{\mathbf{P}}$ in $\overline{\mathbf{P}}^{ \pm}$as in Equations (11a) and (11b).

4. Compute $\Psi_{0}^{ \pm}$according to Equation (14b) and evaluate $\bar{\tau}_{n+1}^{ \pm i}$ as in Equations (15a) and (15b)

5. Backward Euler scheme

$$
\begin{array}{ll}
\operatorname{IF~}\left(\bar{\tau}_{n+1}^{+i}-r_{n}^{+} \leqslant 0\right) \rightarrow & \text { Elastic case } \\
& \dot{r}^{+}=0, r_{n+1}^{+i}=r_{n}^{+}, d_{n+1}^{+i}=d_{n}^{+} \\
\operatorname{ELSE} & \text { Tensile damage } \\
& \dot{r}^{+}=0, r_{n+1}^{+i}=\max \left[r_{n}^{+}, \bar{\tau}_{n+1}^{+i}\right], \dot{d}_{n+1}^{+i}=G^{+}\left(r_{n+1}^{+i}\right) \\
\operatorname{IF}\left(\bar{\tau}_{n+1}^{-i}-r_{n}^{-} \leqslant 0\right) \rightarrow & \text { Elastic case } \\
& \dot{r}^{-}=0, r_{n+1}^{-i}=r_{n}^{-}, d_{n+1}^{-i}=d_{n}^{-} \\
\mathrm{ELSE} \rightarrow & \text { Compressive damage } \\
& \dot{r}^{-}=0, r_{n+1}^{+i}=\max \left[r_{n}^{+}, \bar{\tau}_{n+1}^{+i}\right], \dot{d}_{n+1}^{-i}=G^{+}\left(r_{n}^{-i}\right)
\end{array}
$$

6. OUTPUT: Updated values of the FPK stress vector and tangent constitutive tensor

$$
\begin{aligned}
{\left[\hat{P}_{1}^{\mathrm{m}}\right]_{n+1}^{i} } & =\left(1-d_{n+1}^{+i}\right)\left[\hat{\bar{P}}_{1}^{\mathrm{m}}\right]_{n+1}^{+i}+\left(1-d_{n+1}^{-i}\right)\left[\hat{\bar{P}}_{1}^{\mathrm{m}}\right]_{n+1}^{-i} \\
\mathscr{C}^{\mathrm{mt}} & =\left[\left(1-d^{ \pm}\right) \mathbf{U}^{ \pm} \mathscr{C}^{\mathrm{me}}-G^{ \pm \prime} \hat{\bar{P}}_{1}^{\mathrm{m}} \otimes \hat{\mathbb{L}}^{ \pm}\right]
\end{aligned}
$$

STOP.

fracture energy $G_{\mathrm{f}}^{ \pm}$of the material (obtained from one-dimensional tension or compression tests) and the characteristic length of the fracture zone $l_{\mathrm{c}}$. This calibration ensures mesh-size objective results in numerical simulations $[16,57,58]$, as it will be shown in Section 6 .

From the numerical stand point, a backward Euler scheme is used for the numerical integration of the constitutive damage model. The flow chart with the step-by-step algorithm used in numerical simulations is shown in Table I.

\subsection{Rate-dependent behavior}

Strain rate sensitivity is a widely recognized property of concrete subjected to earthquakes where straining rates as high as $10^{-6} / \mathrm{s}$ can appear (see e.g. [59]). In this work, a viscous regularization of the rate-dependent evolution law for the damage threshold given in Equation (182) is used; however, no regularization is performed on the rate form of damage variables $\dot{d}^{ \pm}$. 
3.2.1. Viscous regularization. The procedure here adopted is inspired in the Perzina regularization for viscoplasticity [60] and the same approach has been previously developed, in the context of the three-dimensional continuum, in the works of References $[42,47,55,56]$, among others. In the present case, Equations (24b) and (24c) have to be replaced by

$$
\begin{gathered}
\dot{r}^{ \pm}=\mu^{ \pm} \phi^{ \pm}\left(\bar{\tau}^{ \pm}, r^{ \pm}\right) \\
\phi^{ \pm}\left(\bar{\tau}^{ \pm}, r^{ \pm}\right)=r_{0}^{ \pm}\left[\frac{\left\langle\bar{\tau}^{ \pm}-r^{ \pm}\right\rangle}{r^{ \pm}}\right]^{a^{ \pm}}
\end{gathered}
$$

where $\phi^{ \pm}$are the viscous damage threshold flow functions. The parameter $\mu^{ \pm}$is termed the fluidity and $a^{ \pm} \in \mathbb{R}^{+}$are material parameters evaluated from one-dimensional tests. It is worth noting that in this case Equations (24a) are rewritten as

$$
\dot{d}^{ \pm}=G^{ \pm \prime} \mu^{ \pm} \phi^{ \pm}\left(\bar{\tau}^{ \pm}, r^{ \pm}\right)
$$

In this manner, the expression previously given in Equation (20) for the dissipation remains unchanged. It has to be also noted that: (i) $\mu^{ \pm} \rightarrow 0$ imply $\dot{d}^{ \pm} \rightarrow 0$ thus a linear-elastic response is obtained; (ii) if $\mu^{ \pm} \rightarrow \infty$ imply $\phi^{ \pm}=\dot{r}^{ \pm} / \mu^{ \pm} \rightarrow 0$ or equivalently $\bar{\tau}^{ \pm} \rightarrow r^{ \pm}$and $\dot{\bar{\tau}}^{ \pm} \rightarrow \dot{r}^{ \pm}$i.e. the rate-independent behavior is recovered. Therefore, the elastic and rate-independent cases constitute limit cases of the proposed regularization.

According to Faria et al. [47], to obtain a mesh-size-independent response for the dynamic loading of mechanical problems involving rate-dependent materials with softening, the parameter $\mu^{ \pm}$must be defined as a function of the characteristic length of the fractured domain, $l_{\mathrm{c}}$. This mesh-size regularization can be achieved using (see Cervera et al. [56] for details)

$$
\mu^{ \pm}=\bar{\mu}^{ \pm}\left[\frac{1}{l_{\mathrm{c}}}-\frac{\left(f_{0}^{ \pm}\right)^{2}}{2 E G_{\mathrm{f}}^{ \pm}}\right] \geqslant 0
$$

where $\bar{\mu}^{ \pm}$are material properties.

3.2.2. Numerical determination of $r^{ \pm}$. The algorithmic updating of the FPK stress vector $\hat{P}_{1}^{\mathrm{m}}$ (see Table I) requires to determine the thresholds $r^{ \pm}$of Equation (28a), preferably in the most closed form as possible. Following standard methods, one can use the generalized mid-point rule as

$$
r_{n+1}^{ \pm}=r_{n}^{ \pm}+\Delta t \mu^{ \pm} \phi^{ \pm}\left(\bar{\tau}_{\alpha}^{ \pm}, r_{\alpha}^{ \pm}\right)
$$

with

$$
\bar{\tau}_{\alpha}^{ \pm}=(1-\alpha) \bar{\tau}_{n}^{ \pm}+\alpha \bar{\tau}_{n+1}^{ \pm}, \quad r_{\alpha}^{ \pm}=(1-\alpha) r_{n}^{ \pm}+\alpha r_{n+1}^{ \pm}
$$

and $\alpha \in\left[\frac{1}{2}, 1\right]$. Rearranging Equation (31) one can construct the following residue to be eliminated:

$$
f\left(r_{n+1}^{ \pm}\right)=\left(r_{n}-r_{n+1}\right)^{ \pm}+\Delta t \mu^{ \pm} r_{0}^{ \pm}\left(\left\langle\bar{\tau}_{\alpha}, r_{\alpha}\right\rangle^{ \pm}\right)^{a^{ \pm}}
$$


In general, one has: (i) if $a^{ \pm} \neq 1$ the problem defined in Equation (33) is non-linear; (ii) if $a^{ \pm} \in\{1,2,3,4\}$ it admits an explicit solution; (iii) in the general case, a Newton-Raphson type of iterative scheme is required to determine $r_{n+1}^{ \pm}$. In this work, an additional simplification has been assumed: $a^{ \pm}=1$ by convenience.

In what regards to the algorithm presented in Table I, Point (5) has to be modified in the following manner:

(i) having obtained $\bar{\tau}_{n+1}^{ \pm(i)}$, compute $\bar{\tau}_{\alpha}^{ \pm(i)}$ according to Equation $\left(32_{1}\right)$.

(ii) Verify $\left(\bar{\tau}_{\alpha}^{ \pm(i)}-r_{n}^{ \pm}\right)<0$; if YES then set: (ii.1) $r_{n+1}^{ \pm}=r_{n}^{ \pm}$and go to (6) of Table I; (ii.2) on the contrary, compute $r_{n+1}^{ \pm(i)}$ using to (33). Evaluate $r_{\alpha}^{ \pm}$according to $\left(32_{2}\right)$. If $\left(\bar{\tau}_{\alpha}^{ \pm}<r_{\alpha}^{ \pm}\right)$ set $r_{n+1}^{ \pm}=r_{n}^{ \pm}$; update the damage $d^{ \pm}=G^{ \pm}$; on the contrary, $d^{ \pm}=G^{ \pm}$and go to (6) of Table I.

3.2.3. Viscous tangent tensor. An advantage of the present viscous regularization is given by the fact that the expression for the tangent relation given in Equation (25) is maintained in the viscous case. It should be noted that, in this case, there are not explicit expressions for $\dot{r}^{ \pm}$as a linear function of $\hat{S}_{n}$.

\section{Remark 3}

Comparing the present formulation with that of [23], some comments can be made: (1) Owing to the fact that there is not a component of viscous stress, viscous secant constitutive tensors are avoided. (2) The linearized increment of the material and co-rotated forms of the FPK stress vector are simply $\Delta \hat{P}_{1}^{\mathrm{m}}=\mathscr{C}^{\mathrm{mv}} \Delta \hat{\mathscr{E}}_{n}$ and $\Delta\left[\hat{P}_{1}\right]=\mathscr{C}^{\mathrm{sv}} \Delta\left[\hat{\varepsilon}_{n}\right]$. Therefore, when linearizing the virtual work principle, the viscous contribution of the tangential stiffness vanishes.

\subsection{Plastic materials}

For the case of materials that can undergo non-reversible deformations, the plasticity model formulated in the material configuration is used for predicting their mechanical response. Assuming small elastic, finite plastic deformations, an adequate form of the free energy density, $\Psi$, and analogous procedures as those for the damage model, we have

$$
\hat{P}_{1}^{\mathrm{m}}=\rho_{0} \frac{\partial \Psi\left(\hat{\mathscr{E}}_{n}^{e}, k_{p}\right)}{\partial \hat{\mathscr{E}}_{n}^{e}}=\mathscr{C}^{\mathrm{ms}}\left(\hat{\mathscr{E}}_{n}-\hat{\mathscr{E}}_{n}^{P}\right)=\mathscr{C}^{\mathrm{me}} \hat{\mathscr{E}}_{n}^{e}
$$

where the $\hat{\mathscr{E}}_{n}^{e}$ is the elastic strain calculated subtracting the plastic strain $\hat{\mathscr{E}}_{n}^{P}$ from the total strain $\hat{\mathscr{E}}_{n}, \rho_{0}$ is the density in the material configuration, $k_{p}$ is the plastic damage internal variable and the material form of the secant constitutive tensor is such that $\mathscr{C}^{\mathrm{ms}}=\mathscr{C}^{\mathrm{me}}$.

Both, the yield function, $\mathscr{F}_{p}$, and plastic potential function, $\mathscr{G}_{p}$, are formulated in terms of the FPK stress vector $\hat{P}_{1}^{\mathrm{m}}$ and the plastic damage internal variable $k_{p}$ as

$$
\begin{aligned}
& \mathscr{F}_{p}\left(\hat{P}_{1}^{\mathrm{m}}, k_{p}\right)=\mathscr{P}_{p}\left(\hat{P}_{1}^{\mathrm{m}}\right)-f_{p}\left(\hat{P}_{1}^{\mathrm{m}}, k_{p}\right)=0 \\
& \mathscr{G}_{p}\left(\hat{P}_{1}^{\mathrm{m}}, k_{p}\right)=K
\end{aligned}
$$


where $\mathscr{P}_{p}\left(\hat{P}_{1}^{\mathrm{m}}\right)$ is the equivalent stress, which is compared with the hardening function $f_{p}\left(\hat{P}_{1}^{\mathrm{m}}, k_{p}\right)$ and $\mathscr{K}$ is a constant value [61]. In this work, $k_{p}$ is a measure of the energy dissipated during the plastic process and, therefore, it is well suited for materials with softening and is defined $[32]$ as

$$
\begin{aligned}
g_{\mathrm{f}}^{P} & =\frac{G_{\mathrm{f}}^{P}}{l_{\mathrm{c}}}=\int_{t=0}^{\infty} \hat{P}_{1}^{\mathrm{m}} \cdot \dot{\mathscr{E}}_{n}^{P} \mathrm{~d} t \\
0 & \leqslant\left[k_{p}=\frac{1}{g_{\mathrm{f}}^{P}} \int_{t=0}^{t} \hat{P}_{1}^{\mathrm{m}} \cdot \dot{\mathscr{E}}_{n}^{P} \mathrm{~d} t\right] \leqslant 1
\end{aligned}
$$

where $G_{\mathrm{f}}^{P}$ is the specific plastic fracture energy density of the material in tension and $l_{\mathrm{c}}$ is the length of the fractured domain defined in analogous manner as for the damage model. The integral term in Equation (36b) corresponds to the energy dissipated by means of plastic work.

The flow rules for the internal variables $\hat{\mathscr{E}}_{n}^{P}$ and $k_{p}$ are defined as

$$
\begin{aligned}
\dot{\hat{\mathscr{E}}}_{n}^{P} & =\dot{\lambda} \frac{\partial \mathscr{G}_{p}}{\partial \hat{P}_{1}^{\mathrm{m}}} \\
\dot{k}_{p} & =\dot{\lambda} \hat{\varrho}\left(\hat{P}_{1}^{\mathrm{m}}, k_{p}, G_{\mathrm{f}}^{P}\right) \cdot \frac{\partial \mathscr{G}_{p}}{\partial \hat{P}_{1}^{\mathrm{m}}}=\hat{\varrho}\left(\hat{P}_{1}^{\mathrm{m}}, k_{p}, G_{\mathrm{f}}^{P}\right) \cdot \dot{\hat{\mathscr{E}}}_{n}^{P}
\end{aligned}
$$

where $\dot{\lambda}$ is the plastic consistency parameter and $\hat{\varrho}$ is the hardening vector as described in References $[16,61]$. In what regards the hardening function of Equation (35a), the following evolution equation has been proposed:

$$
f_{p}\left(\hat{P}_{1}^{\mathrm{m}}, k_{p}\right)=r \sigma_{t}\left(k_{p}\right)+(1-r) \sigma_{\mathrm{c}}\left(k_{p}\right)
$$

where $r \in \mathbb{R}$ considers the relation existing between components of the stress tensor in compression and tension, and it can be consulted in [16]. The scalar functions $\sigma_{t}\left(k_{p}\right)$ and $\sigma_{\mathrm{c}}\left(k_{p}\right)$ describe the evolution of the yielding threshold in uniaxial tension and compression tests, respectively [61].

As it is a standard practice in plasticity, the loading/unloading conditions are derived in the standard form from the Kuhn--Tucker relations formulated for problems with unilateral restrictions, i.e. (a) $\dot{\lambda} \geqslant 0$, (b) $\mathscr{F}_{p} \leqslant 0$ and (c) $\dot{\lambda} \mathscr{F}_{p}=0$. Starting from the plastic consistency condition $\dot{\mathscr{F}}_{p}=0$ and considering the flow rules it is possible to deduce the explicit form of $\dot{\lambda}$ as $[16,61]$

$$
\dot{\lambda}=-\frac{\frac{\partial \mathscr{F} p}{\partial \hat{P}_{1}^{\mathrm{m}}} \cdot\left(\mathscr{C}^{\mathrm{me}} \dot{\mathscr{\mathscr { E }}}_{n}\right)}{\left\{\frac{\partial \mathscr{F}_{p}}{\partial \hat{P}_{1}^{\mathrm{m}}} \cdot\left(\mathscr{C}^{\mathrm{me}} \frac{\partial \mathscr{G}_{p}}{\partial \hat{P}_{1}^{\mathrm{m}}}\right)-\frac{\partial f_{p}}{\partial k_{p}} \hat{\underline{Q}} \cdot \frac{\partial \mathscr{G}_{p}}{\partial \hat{P}_{1}^{\mathrm{m}}}\right\}}
$$

The material form of the tangent constitutive tensor is calculated taking the time derivative of Equation (34), considering the flow rule of Equation (37b) and the plastic consistency parameter 
of Equation (39) as

$$
\delta \hat{P}_{1}^{\mathrm{m}}=\mathscr{C}^{\mathrm{mt}} \delta \hat{\mathscr{E}}_{n}=\left[\mathscr{C}^{\mathrm{me}}-\frac{\left(\mathscr{C}^{\mathrm{me}} \frac{\partial \mathscr{G}_{p}}{\partial \hat{P}_{1}^{\mathrm{m}}}\right) \otimes\left(\mathscr{C}^{\mathrm{me}} \frac{\partial \mathscr{F}_{p}}{\partial \hat{P}_{1}^{\mathrm{m}}}\right)}{\frac{\partial \mathscr{F}_{p}}{\partial \hat{P}_{1}^{\mathrm{m}}} \cdot\left(\mathscr{C}^{\mathrm{me}} \frac{\partial \mathscr{G}_{p}}{\partial \hat{P}_{1}^{\mathrm{m}}}\right)-\frac{\partial \mathscr{F}_{p}}{\partial k_{p}} \hat{\varrho} \cdot\left(\frac{\partial \mathscr{G}_{p}}{\partial \hat{P}_{1}^{\mathrm{m}}}\right)}\right] \delta \hat{\mathscr{E}}_{n}
$$

\subsection{Mixing theory for composites}

Each material point on the beam cross section is treated as a composite material according to the mixing theory [31,61]. This theory is based on the early works of Truesdell and Toupin [62] for bi-phasic materials and further exploited by a number of authors (see e.g. Ortiz and Popov [30] and references therein, among many others).

In this theory, the interaction between all the components defines the overall mechanical behavior of the composite at material point level. Supposing $N$ different components coexisting in a generic material point subjected to the same material strain field $\hat{\mathscr{E}}_{n}$, we have the following closing equation: $\hat{\mathscr{E}}_{n} \equiv\left(\hat{\mathscr{E}}_{n}\right)_{1}=\cdots=\left(\hat{\mathscr{E}}_{n}\right)_{q}=\cdots=\left(\hat{\mathscr{E}}_{n}\right)_{N}$, which imposes the strain compatibility between components. The free energy density of the composite, $\bar{\Psi}$, is obtained as the weighted sum of the free energy densities of the $N$ components. The weighting factors, $k_{q}$, correspond to the quotient between the volume of the $q$ th component, $V_{q}$, and the total volume, $V$, such that $\sum_{q} k_{q}=1$.

The material form of the FPK stress vector $\hat{P}_{1}^{\mathrm{mt}}$ for the composite, including the participation of rate-dependent effects, is obtained in analogous way as for simple materials i.e.

$$
\hat{P}_{1}^{\mathrm{mt}} \equiv \sum_{q}^{N} k_{q}\left(\hat{P}_{1}^{\mathrm{m}}+\hat{P}_{1}^{\mathrm{mv}}\right)_{q}=\sum_{q}^{N} k_{q}\left[(1-d) \mathscr{C}^{\mathrm{me}}\left(\hat{\mathscr{E}}_{n}+\frac{\eta}{E_{0}} \hat{\mathscr{S}}_{n}\right)\right]_{q}
$$

where $\left(\hat{P}_{1}^{\mathrm{m}}\right)_{q}$ and $\left(\hat{P}_{1}^{\mathrm{mv}}\right)_{q}$ correspond to the rate-independent and -dependent stresses of each one of the $N$ components, respectively. The material form of the secant and tangent constitutive tensors for the composite, $\overline{\mathscr{C}}^{\mathrm{ms}}$ and $\overline{\mathscr{C}}^{\mathrm{mt}}$, is obtained as $[16,23,61]$

$$
\overline{\mathscr{C}}^{\mathrm{ms}} \equiv \sum_{q=1}^{N} k_{q}\left(\mathscr{C}^{\mathrm{ms}}\right)_{q}, \quad \overline{\mathscr{C}}^{\mathrm{mt}} \equiv \sum_{q=1}^{N} k_{q}\left(\mathscr{C}^{\mathrm{mt}}\right)_{q}
$$

where $\left(\mathscr{C}^{\mathrm{ms}}\right)_{q}$ and $\left(\mathscr{C}^{\mathrm{mt}}\right)_{q}$ are the material form of the secant and tangent constitutive tensors of the $q$ th component.

\subsection{Constitutive relations for EDDs}

The constitutive law proposed for EDDs is based on a previous work of the authors [26] which provides a versatile strain-stress relationship with the following general form:

$$
P_{d}^{\mathrm{m}}\left(\mathscr{E}_{d_{1}}, \dot{\mathscr{E}}_{d_{1}}, t\right)=P_{d_{1}}^{\mathrm{m}}\left(\mathscr{E}_{d_{1}}, t\right)+P_{d_{2}}^{\mathrm{m}}\left(\dot{\mathscr{E}}_{d_{1}}, t\right)
$$


where $P_{d}^{\mathrm{m}}$ is the average stress in the EDD, $\mathscr{E}_{d_{1}}$ the strain level, $t$ the time, $\dot{\mathscr{E}}_{d_{1}}$ the strain rate and, $P_{d_{1}}^{\mathrm{m}}$ and $P_{d_{2}}^{\mathrm{m}}$ are the strain-dependent and rate-dependent parts of the total stress in the device, respectively.

The model uncouples the total stress in viscous and non-viscous components, which correspond, in terms of rheological models, to a viscous dashpot device acting in parallel with a non-linear hysteretic spring. The purely viscous component does not requires to be a linear function of the strain rate.

From the results obtained from experiments carried out on a large variety of different types of devices, it is possible to see that the function $P_{d_{1}}^{\mathrm{m}}$ should have the following characteristics:

(i) Hardening for strain levels over $150 \%$.

(ii) Variable instantaneous stiffness.

(iii) For elastomer-based devices, the initial slope of a given loading or unloading branch of their characteristic force-displacement curves is a function of the point in the strain-stress space where the velocity of deformation changes of sign (see [26]).

3.5.1. Rate-dependent part. The viscous component of the stress has the following form:

$$
P_{d_{2}}^{\mathrm{m}}\left(\dot{\mathscr{E}}_{d_{1}}, t\right)=c_{d}\left(\dot{\mathscr{E}}_{d_{1}}\right) \dot{\mathscr{E}}_{d_{1}}
$$

where $c_{d}$ is the (non-linear) viscous coefficient function of the device that is obtained fitting a polynomial to experimental data. A method is proposed in Reference [26] for the case of elastomerbased devices; however, the same procedure can be applied to other base materials.

3.5.2. Rate-independent part. The capacity of the model for simulating hardening for strain levels over $150 \%$ (e.g. in the case of elastomer-based devices) is given by an adequate non-linear-elastic backbone added to the non-viscous hysteretic cycles, as it can be seen in the scheme of Figure 5. The proposed backbone is defined numerically by means of a polynomial, whose coefficients are fitted to experimental data; for example, for the case of the high damping elastomer of Reference [26]

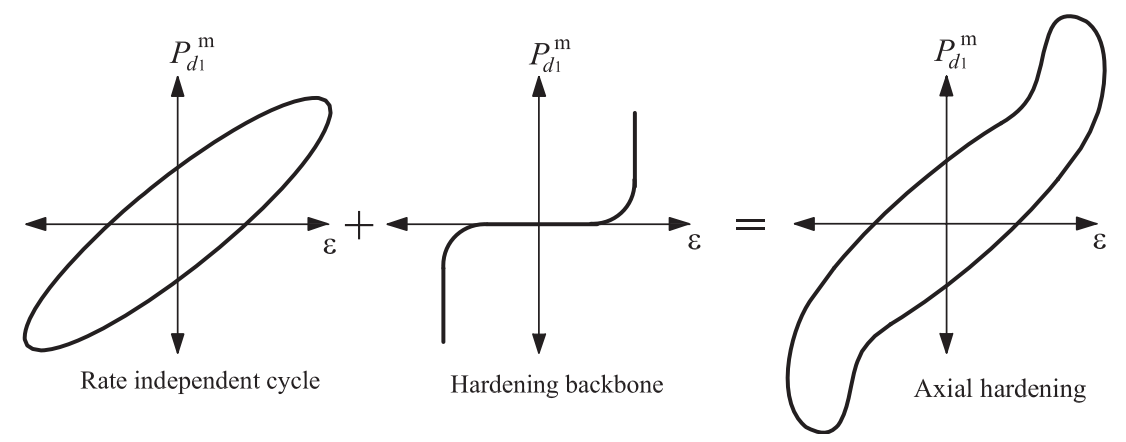

Figure 5. Non-linear elastic backbone added to the rate-independent part of the constitutive relation. 
the following formula is obtained: $P_{d_{1}}^{\mathrm{m} h}=\operatorname{sgn}\left[\dot{\mathscr{E}}_{d_{1}}\right] A_{0}\left(\left\langle\left|\mathscr{E}_{d_{1}}\right|-A_{1}\right\rangle\right)^{A_{2}}$, where $A_{0}, A_{1}$ and $A_{2}$ are scalars obtained from experimental tests.

The response of the non-linear hysteretic spring is obtained solving the following system of non-linear differential equations:

$$
\begin{aligned}
P_{d_{1}}^{\mathrm{m}}\left(\mathscr{E}_{d_{1}}, t\right) & =K_{\mathrm{y}}\left(\mathscr{E}_{d_{1}}^{b_{1}}, P_{d}^{\mathrm{mb}}\right) \mathscr{E}_{d_{1}}+\left[K_{e}\left(\mathscr{E}_{d_{1}}^{\mathscr{b}^{b}}, P_{d}^{\mathrm{mb}}\right)-K_{\mathrm{y}}\left(\mathscr{E}_{d_{1}}^{\mathrm{b}}, P_{d}^{\mathrm{mb}}\right)\right] e \\
\text { if } \dot{\mathscr{E}}_{d_{1}} e \geqslant 0 \rightarrow \dot{e}= & {\left[1-\left|\frac{e}{d_{\mathrm{y}}\left(\mathscr{E}_{d_{1}}^{\mathrm{b}}, P_{d}^{\mathrm{mb}}\right)}\right|^{n\left(\mathscr{E}_{d_{1}}^{\mathrm{b}}, P_{d}^{\mathrm{mb}}\right)}\right] \dot{\mathscr{E}}_{d_{1}} } \\
\text { else } \rightarrow \dot{e} & =\dot{\mathscr{E}}_{d_{1}}
\end{aligned}
$$

where $K_{\mathrm{y}}$ is the post yielding stiffness, $K_{e}$ the elastic stiffness, $d_{\mathrm{y}}$ is the yielding strain of the material and $e$ represents an internal variable of plastic (hysteretic) strain, which takes values in the range $\left[-d_{\mathrm{y}}, d_{\mathrm{y}}\right]$. The parameter $n$ in the associated flow rule of Equation (45c) describes the degree of smoothness exhibited by the transition zone between the pre and the post yielding branches of the hysteretic cycle. The solution procedure solves the system of equations taking into account that $K_{e}, K_{\mathrm{y}}, d_{\mathrm{y}}$, and $n$ are function of the point in the strain-stress space where the last change of sign of the strain rate has occurred, which is denoted by $\left(\mathscr{E}_{d_{1}}^{\mathrm{b}}, P_{d}^{\mathrm{mb}}\right)$ in Equations (45a) and (45c). Therefore, the proposed algorithm (see [26]) updates the parameters of the model for each change of sign of the strain rate. If there is no change in the sign of the strain rate, the parameters of the model are maintained constants. Hardening can be incorporated by means of adding the non-linear elastic backbone $P_{d_{1}}^{\mathrm{m} h}$ to Equation (45a).

The parameters $K_{e}, K_{\mathrm{y}}, d_{\mathrm{y}}, n$ are non-linear functions of $\left(\mathscr{E}_{d_{1}}^{\mathrm{b}}, P_{d}^{\mathrm{mb}}\right)$, i.e.

$$
K_{e}=\wp_{1}\left(\mathscr{E}_{d_{1}}^{\mathrm{b}}, P_{d}^{\mathrm{mb}}\right), \quad K_{\mathrm{y}}=\wp_{2}\left(\mathscr{E}_{d_{1}}^{\mathrm{b}}, P_{d}^{\mathrm{mb}}\right), \quad d_{\mathrm{y}}=\wp_{3}\left(\mathscr{E}_{d_{1}}^{\mathrm{b}}, P_{d}^{\mathrm{mb}}\right), \quad n=\wp_{4}\left(\mathscr{E}_{d_{1}}^{\mathrm{b}}, P_{d}^{\mathrm{mb}}\right)
$$

Explicit expression for $\wp_{k}(k=1, \ldots, 4)$ (in function of $\left.\left(\mathscr{E}_{d_{1}}^{\mathrm{b}}, P_{d}^{\mathrm{mb}}\right)\right)$ are determined from experimental data. In any case, it is possible to simulate the mechanical behavior of a wide variety of devices, e.g.

- $c_{d}=0, \wp_{1}=\wp_{2}=$ constant, $\wp_{3} \sim \infty$ and $\wp_{4}=1$ : an elastic spring is obtained. This case corresponds to devices designed as re-centering ${ }^{\ddagger \ddagger}$ elements in structures.

- $c_{d}=$ constant, $\wp_{1}=\wp_{2}=0, \wp_{3} \sim \infty$ and $\wp_{4}=1:$ a viscous dashpot is obtained. This case can be found in devices applied to control the effects of wind loads.

- $c_{d}=$ constant, $\wp_{1}=\wp_{2}=$ constant, $\wp_{3} \sim \infty$ and $\wp_{4}=1$ : Maxwell's model is obtained. This case corresponds to a typical viscous device with re-centering mechanism. See Figure 6(a) where the loading was defined by sinusoidal path of imposed strains with increasing amplitude up to a maximum value of $2 \mathrm{~mm} / \mathrm{mm}$ (the same applies for the rest of the figures).

\footnotetext{
${ }^{\ddagger}$ Re-centering forces are considered to be of importance in structures subjected to strong earthquakes. Re-centering mechanisms help to recover the original configuration of the structure after the seismic action.
} 


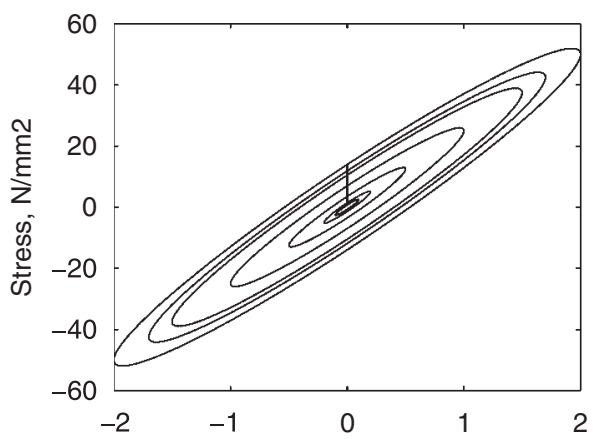

(a)
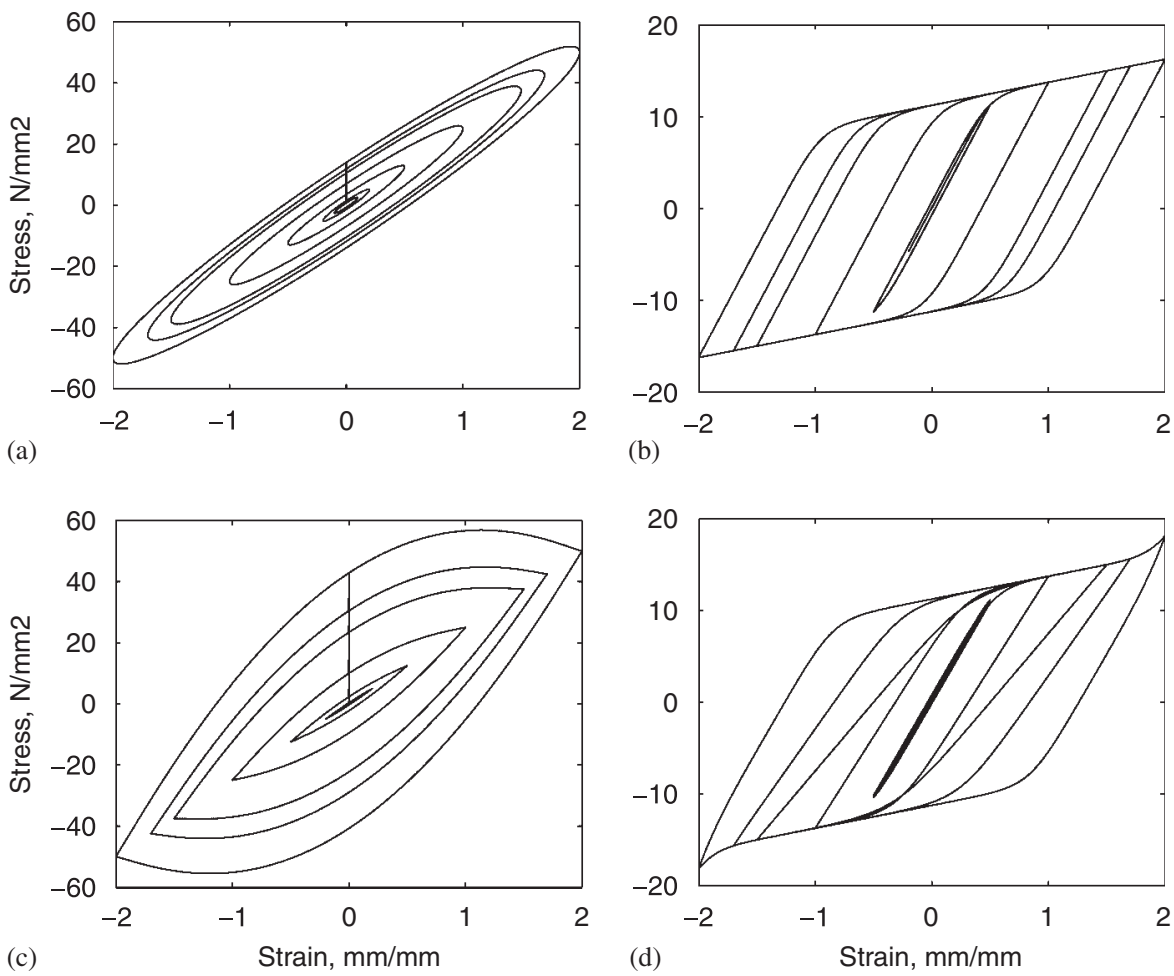

Figure 6. Examples of EDD's behaviors: (a) Maxwell model, $c_{d}=7, \wp_{1}=\wp_{2}=25, \wp_{3} \sim \infty$ and $\wp_{4}=1$; (b) bilinear inviscid plastic model, $c_{d}=0, n=1, \wp_{1}=25, \wp_{2}=2.5$ and $\mathscr{E}$ y $=0.5$; (c) non-linear dashpot, $c_{d}=7, K_{\mathrm{c}}=2.1, \wp_{1}=\wp_{2}=25, \wp_{3} \sim \infty$ and $\wp_{4}=1$; and (d) rubber model, $\wp_{1}=-34.9267+217.7 \mathscr{E}_{d_{1}}-530.1\left(\mathscr{E}_{d_{1}}\right)^{2}+655.1\left(\mathscr{E}_{d_{1}}\right)^{3}-451.3\left(\mathscr{E}_{d_{1}}\right)^{4}+176.7\left(\mathscr{E}_{d_{1}}\right)^{5}$

$-39.7\left(\mathscr{E}_{d_{1}}\right)^{6}+26.6\left(\mathscr{E}_{d_{1}}\right)^{7}, \wp_{2}=2.5, P_{d_{1}}^{\mathrm{m} h}=\operatorname{sign}\left(\mathscr{E}_{d_{1}}^{b}\right) * 8 *\left(\left|\mathscr{E}_{d_{1}}^{b}\right|-1.25\right)^{5}\left(\right.$ if $\left.\mathscr{E}_{d_{1}}^{b}>1.35\right)$.

- $c_{d}=$ constant, $\wp_{1} \geqslant \wp_{2}>0, n \in[1,100]$ and $\mathscr{E}_{\mathrm{y}}>0$ : a visco-plastic device can be simulated (assuming uncoupled Maxwell's viscosity). Particularly, if $c_{d}=\wp_{2}=0$ and $n=1$ a bilinear perfectly plastic model is obtained. See Figure 6(b).

- $c_{d}=\left(\dot{\mathscr{E}}_{d_{1}}\right)^{K_{\mathrm{c}}}\left(0<K_{\mathrm{c}}<1\right), \wp_{1}=\wp_{2}=0, \wp_{3} \sim \infty$ and $\wp_{4}=1$ : a non-linear viscous dashpot is obtained. Some modern viscous devices employ modern valves's systems that produces the non-linear force-velocity curve with a saturation level (see e.g. [4] Chapter 6 and Figure 6(c).

- In the case of devices made of high damping elastomers (such as those considered in Reference [26]) it is possible to consider: $c_{d}=$ constant, $\wp_{1}=\sum_{k=0}^{N_{\mathrm{c}}} A_{k}\left(\mathscr{E}_{d_{1}}\right)^{k}, A_{k}=$ constant, $\forall k=\left(1 \ldots N_{\mathrm{c}}\right)$; the post-yielding stiffness function, $\wp_{2}$, is maintained constant. The analytical expression of $\wp_{3}$ is $\wp_{3}=\left(\left|P_{d}^{\mathrm{mb}}\right|-\left|P_{0}^{\mathrm{mb}}-P_{d}^{\mathrm{mb}}\right|\right) /\left(\wp_{2}-\wp_{1}\right)$; where $P_{0}^{\mathrm{mb}}$ is a parameter calculated evaluating the line with slope $\wp_{2}$ at zero strain level. The function $\wp_{4}$ can be taken $\wp_{4} \approx 1$ (see Figure 6(d)).

More complex material behaviors, such as those of other types of rubber or smart-based devices, can be simulated by means of providing suitable functions for the parameters of the model (see Equation (46)). 


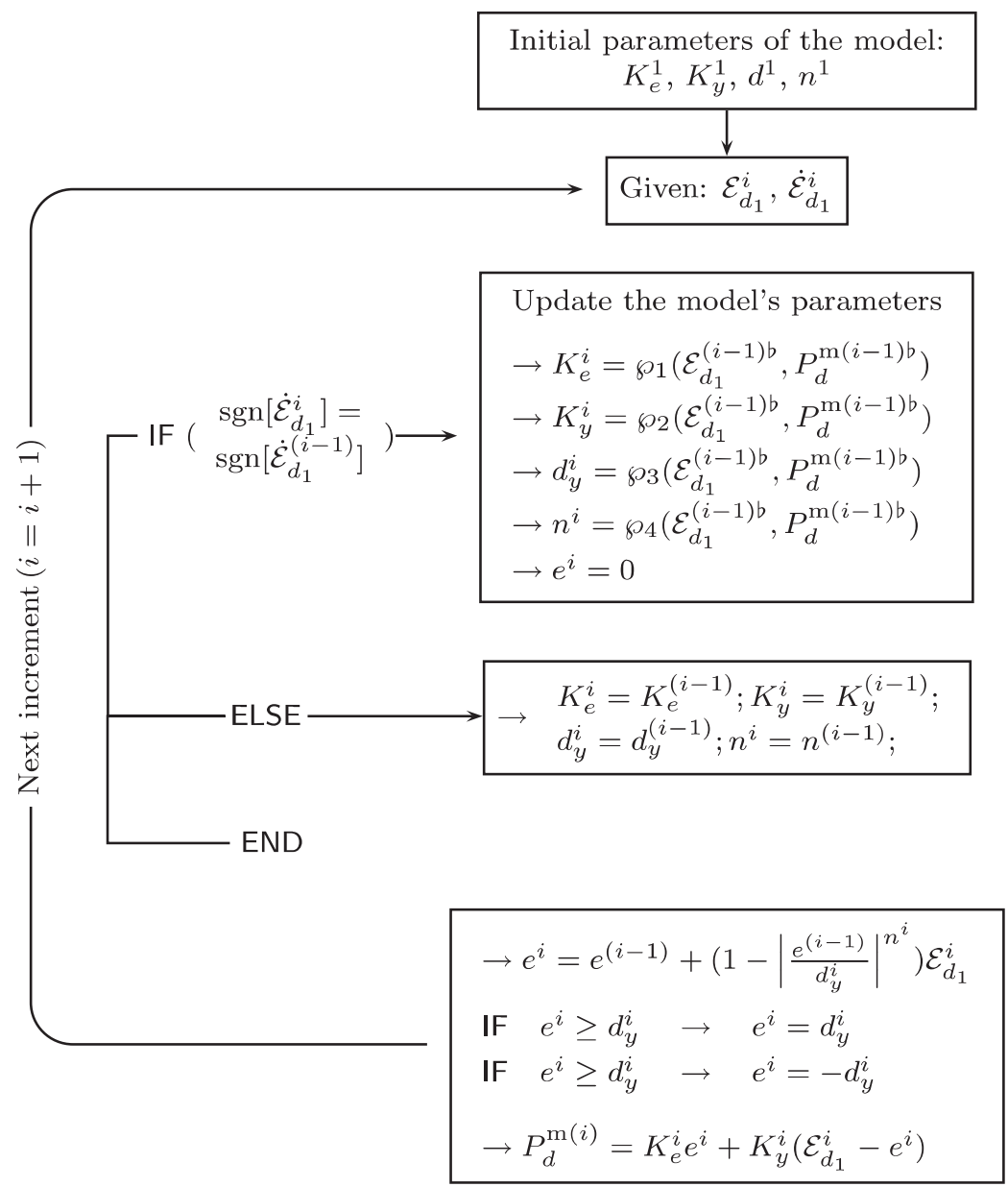

Figure 7. Flowchart of the constitutive relation for EDDs.

3.5.3. Tangent stiffness. The tangent relation for the EDDs is obtained numerically using the perturbation method [63]. It consists in applying a small increment ${ }^{\S \S}$ to $\mathscr{E}_{d_{1}}$, which is denoted by $\Delta \mathscr{E}_{d_{1}}$; and after solving the system of Equation (45c) for the total strain $\left(\mathscr{E}_{d_{1}}+\Delta \mathscr{E}_{d_{1}}\right)$, the new stress level $P_{d_{1}}^{\mathrm{m}}$ is determined. Furthermore, the hardening and viscous contributions have to be added to obtain $P_{d}^{\mathrm{m}}\left(\mathscr{E}_{d_{1}}+\Delta \mathscr{E}_{d_{1}}\right)$. The tangential stiffness of the device, $K^{t}$, is then calculated as

$$
K^{t}=\frac{\Delta P_{d}^{\mathrm{m}}}{\Delta \mathscr{E}_{d_{1}}}=\frac{P_{d}^{\mathrm{m}}\left(\mathscr{E}_{d_{1}}+\Delta \mathscr{E}_{d_{1}}\right)-P_{d}^{\mathrm{m}}\left(\mathscr{E}_{d_{1}}\right)}{\Delta \mathscr{E}_{d_{1}}}
$$

\footnotetext{
$\overline{\S \& \text { Here the notion }}$ of smallness corresponds to the precision of the machine used in the numerical simulations. 
It is important to note that the sign of the perturbation has to be the same as $\dot{\mathscr{E}}_{d_{1}}$ in order to obtain a tangential stiffness consistent with the sign of the loading process in the device, when cyclic actions are considered.

3.5.4. Integration algorithm. The numerical algorithm starts by assigning an initial values to the parameters of the model. For each strain level, $\mathscr{E}_{d_{1}}^{i}$, the algorithm verifies if the strain rate changes of sign. If this is the case, an updating procedure for the parameters $\wp_{k}(k=1, \ldots, 4)$ is carried out. On the contrary, the parameters are maintained. Then, plastic strain, stress and the tangential relation are estimated. The flow-chart of the algorithm is shown in Figure 7.

\section{NUMERICAL IMPLEMENTATION}

In order to obtain a Newton-type numerical solution, the linearized part of the weak form of Equation (7) is required, which can be written as

$$
\mathscr{L}\left[G_{w}\left(\hat{\varphi}_{*}, \boldsymbol{\Lambda}_{*}, \hat{h}\right)\right]=G_{w}\left(\hat{\varphi}_{*}, \boldsymbol{\Lambda}_{*}, \hat{h}\right)+D G_{w}\left(\hat{\varphi}_{*}, \boldsymbol{\Lambda}_{*}, \hat{h}\right) \cdot \hat{p}
$$

where $\mathscr{L}\left[G_{w}\left(\hat{\varphi}_{*}, \Lambda_{*}, \hat{h}\right)\right]$ is the linear part of the functional $G_{w}(\hat{\varphi}, \boldsymbol{\Lambda}, \hat{h})$ at the configuration defined by $(\hat{\varphi}, \boldsymbol{\Lambda})=\left(\hat{\varphi}_{*}, \boldsymbol{\Lambda}_{*}\right)$ and $\hat{p} \equiv(\Delta \hat{\varphi}, \Delta \hat{\theta})$ is an admissible variation. The term $G_{w *}$ supplies the unbalanced force vector obtained adding the contributions of the inertial, external and internal terms; and $D G_{w *} \cdot \hat{p}$, gives the tangential stiffness [27].

On the one hand, the linearization of the inertial and external components, $G_{w}^{\text {ine }}$ and $G_{w}^{\text {ext }}$, yields the inertial and load-dependent parts of the tangential stiffness, $K_{\mathrm{I} *}$ and $K_{\mathrm{P} *}$, respectively; and they can be consulted in $[25,27]$. On the other hand, the linearization of the internal term is obtained as $[16,23]$

$$
D G_{w *}^{\mathrm{int}} \cdot \hat{p}=\int_{0}^{L} \hat{h}^{\mathrm{T}}\left(\mathbf{B}_{*}^{\mathrm{T}} \Delta \Phi-\mathbf{n}_{S *} \hat{p}\right) \mathrm{d} S
$$

where $\Delta \Phi^{\mathrm{T}}=\left[\Delta \hat{n}_{*}^{\mathrm{T}} \Delta \hat{m}_{*}^{\mathrm{T}}\right]$, the operator $\mathbf{n}_{S *}$ contributes to the geometric part of the tangent stiffness and $\mathbf{B}_{*}$ relates $\hat{h}$ and the co-rotated variation of the strain vectors. Explicit expression for $\mathbf{B}$ and $\mathbf{n}_{S *}$ can be found in References [16, 27, 28], respectively.

The computation of $\Delta \Phi$ appearing in Equation (49) requires taking into account the linearized relation between $\hat{P}_{1}^{m}$ and $\hat{\mathscr{E}}_{n}$. After integrating over the beam cross section, the following result is obtained:

$$
\Delta \Phi=\left(\mathbf{C}^{\mathrm{sv}} \mathbf{B}_{*}-\widetilde{\mathbf{F}}\right) \hat{p}
$$

where $\mathbf{C}^{\mathrm{sv}}$ is the spatial form of the cross-sectional tangent constitutive tensor depending on the material properties and $\widetilde{\mathbf{F}}$ is the stress-dependent tensor (see [16,23] for details). Finally, Equation (50) allows to rewrite Equation (49) as

$$
D G_{w}^{\mathrm{int}} \cdot p=\int_{0}^{L} \hat{h}^{\mathrm{T}} \mathbf{B}_{*}^{\mathrm{T}} \mathbf{C}_{*}^{\mathrm{st}} \mathbf{B}_{*} \hat{p} \mathrm{~d} S+\int_{0}^{L} \hat{h}^{\mathrm{T}}\left(\widetilde{\mathbf{n}}_{S *}-\mathbf{B}_{*}^{\mathrm{T}} \widetilde{\mathbf{F}}_{*}\right) \hat{p} \mathrm{~d} S=K_{\mathrm{M} *}+K_{\mathrm{G} *}
$$


where $K_{\mathrm{G} *}, K_{\mathrm{M} *}$, evaluated at $\left(\hat{\varphi}_{*}, \Lambda_{*}\right)$, give the geometric and material parts of the tangent stiffness, which allows to rewrite Equation (48) as

$$
\mathscr{L}\left[\mathrm{G}_{*}\right]=G_{w *}+K_{\mathrm{I} *}+K_{\mathrm{M} *}+K_{\mathrm{V} *}+K_{\mathrm{G} *}+K_{\mathrm{P} *}
$$

The solution of the discrete form of Equation (51) by using the finite element method follows an identical procedure as that described in [27] for an iterative Newton-Raphson integration scheme and it will not be discussed here.

Newmark's implicit time-stepping algorithm has been chosen as integration method following the development originally proposed by Simo and Vu-Quoc [25]. For the rotational part, the time-stepping procedure takes place in $\mathrm{SO}(3)$ and the basic steps, as well as the iterative update algorithm for the strain and strain rate vectors, are given in Reference [23].

\section{Remark 4}

Recently, the attention of the research community has been directed toward energy-momentumconserving time-stepping schemes. Some algorithms has been developed for inelastic materials inheriting conservative properties in the elastic range. In particular, Armero [64] presents an algorithm for multiplicative plasticity at finite strains that recovers the energy-momentum-conserving properties in the elastic range. In Reference [65] Noels et al., propose a modification of the variational updates framework to introduce numerical dissipation for high-frequency modes, leading to a energy-dissipative momentum-conserving algorithm for elastoplasticity. In the case of elastic structural elements with large rotations, it is possible to consult [66] and more recently [67]; however, in this last case, the conservative properties do not have been extended to the inelastic range.

\section{CROSS-SECTIONAL ANALYSIS AND DAMAGE INDICES}

\subsection{Cross-sectional analysis}

The cross-sectional analysis is carried out by expanding each integration point of the beam axis in a set of integration points located onto the cross section. Then, the cross sections are meshed into a grid of quadrilaterals, each of them corresponding to a fiber oriented along the beam axis. The geometry of each quadrilateral is described by means of normalized bi-dimensional shape functions and several integration points can be specified according to a specific integration rule. The average value of a quantity, [·], for example the components of FPK stress vector or the tangential tensor existing on a quadrilateral, are

$$
[\cdot]=\frac{1}{A_{\mathrm{c}}} \int[\cdot] \mathrm{d} A_{\mathrm{c}}=\frac{1}{A_{\mathrm{c}}} \sum_{p=1}^{N_{p}} \sum_{q=1}^{N_{q}}[\cdot]\left(y_{p}, z_{q}\right) J_{p_{q}} W_{p_{q}}
$$

where $A_{\mathrm{c}}$ is the area of the quadrilateral, $N_{p}$ and $N_{q}$ are the number of integration points in the two directions of the normalized geometry, $[\cdot]\left(y_{p}, z_{q}\right)$ is the value of the quantity [.] existing on a integration point with coordinates $\left(y_{p}, z_{q}\right)$ with respect to the reference beam axis, $J_{p q}$ is the Jacobian of the transformation between normalized coordinates and cross-sectional coordinates and $W_{p q}$ are the weighting factors. Two additional integration loops are required. The first one runs over the quadrilaterals and the second loop runs over each simple material associated with the composite of the quadrilateral. More details can be consulted in [16, 23]. 


\subsection{Damage indices}

A measure of the damage level of a material point can be obtained as the ratio of the existing stress level to its elastic counterpart. Following this idea, it is possible to define the fictitious damage variable $\check{D}$ as [51]

$$
\sum_{i=1}^{3}\left|P_{1 i}^{\mathrm{m}}\right|=(1-\check{D}) \sum_{i=1}^{3}\left|P_{1 i 0}^{\mathrm{m}}\right| \rightarrow \check{D}=1-\frac{\sum_{i=1}^{3}\left|P_{1 i}^{\mathrm{m}}\right|}{\sum_{i=1}^{3}\left|P_{1 i 0}^{\mathrm{m}}\right|}
$$

where $\left|P_{1 i}^{\mathrm{m}}\right|$ and $\left|P_{1 i 0}^{\mathrm{m}}\right|$ are the absolute values of the components of the existing and elastic stress vectors, respectively. Initially, the material remains elastic and $\check{D}=0$, but when all the energy of the material has been dissipated $\left|P_{1 i}^{\mathrm{m}}\right| \rightarrow 0$ and $\check{D} \rightarrow 1$. Equation (54) can be extended to consider elements or even the whole structure by means of integrating over a finite volume as follows:

$$
\check{D}=1-\frac{\int_{V_{p}}\left(\sum_{i}\left|P_{1 i}^{\mathrm{m}}\right|\right) \mathrm{d} V_{p}}{\int_{V_{p}}\left(\sum_{i}\left|P_{1 i 0}^{\mathrm{m}}\right|\right) \mathrm{d} V_{p}}
$$

where $V_{p}$ is the volume of the part of the structure. Equation (55) is easily implemented in an standard finite element method code without requiring large extra memory storage.

\section{NUMERICAL EXAMPLES}

\subsection{Mesh-independent response}

The cantilever beam of Figure 8 is used to verify if regularizating the constitutive equations according to the fracture energy and the characteristic length of the fractured zone, a meshsize-independent response is obtained for softening materials. Additionally, a global mechanical response independent of the cross-sectional discretization is verified.

The beam is made of a degrading material simulated using the tension-compression model described in Section 3.1. The mechanical properties are: (i) $E=1.5 \times 10^{5} \mathrm{Nmm}^{-2}$; (ii) $v=0.1$;
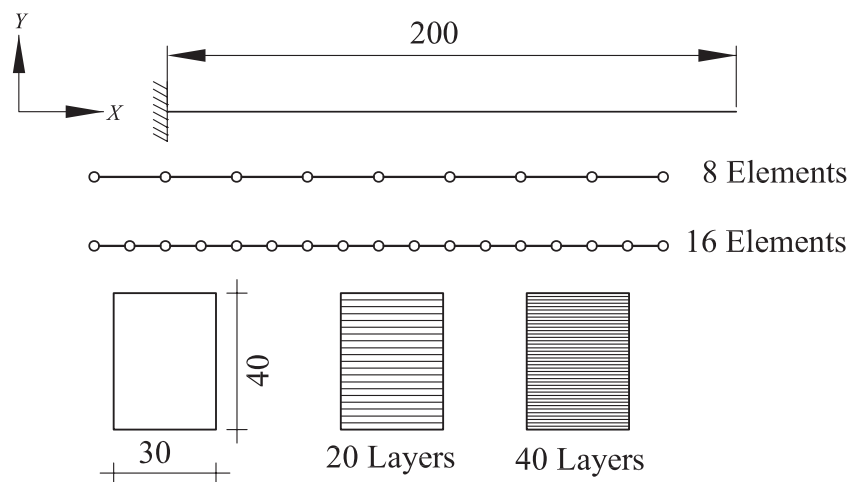

Figure 8. Cantilever beam. 

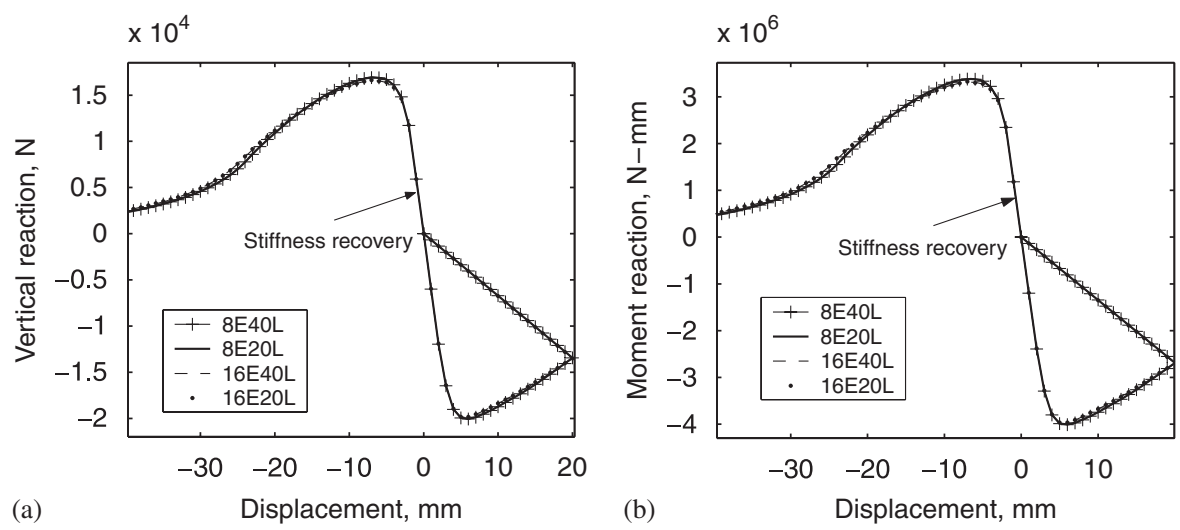

Figure 9. Mesh-independent response: (a) vertical displacement versus vertical reaction and (b) vertical displacement versus moment reaction.

(iii) $f_{0}^{-}=500 \mathrm{Nmm}^{-2}$; (iv) $f_{0}^{+}=250 \mathrm{Nmm}^{-2} ;$ (v) $G_{\mathrm{f}}^{-}=500 \mathrm{Nmm}^{-2} \quad\left(G_{\mathrm{f}}^{+}=125 \mathrm{Nmm}^{-2}\right)$; (vi) $K=1$. An imposed displacement was applied on the free end in the $Y$ direction. The loading path was the following: 20 steps of $1 \mathrm{~mm}$ and then 60 steps of the same magnitude in the opposite direction.

Four discretizations were used: (1) Eight linear elements along the beam axis and 20 layers in the cross section. This case is denoted as 8E20L; (2) Eight linear elements along the beam axis and 40 layers in the cross section (case 8E40L); (3) Sixteen linear elements along the beam axis and 20 layers in the cross section (case 16E20L); (4) Sixteen linear elements along the beam and 40 layers in the cross section (case 16E40L) (see Figure 8). The Gauss integration rule was considered in the simulations. The length of the fractured zone corresponds to the characteristic length associated with the integration point.

Figures 9(a) and (b) show two capacity curves relating the vertical reaction and moment reaction with the vertical displacement of the free end, respectively. It is possible to see that the numerical responses are almost the same for the four cases considered, confirming thus the independency of the global mechanical response with the number of elements and the specific discretization of the cross section. It is possible to appreciate the fact that the independency between tensile and compressive degradations produces the stiffness recovery phenomenon in the global response when reverse loading paths are applied.

Figures 10(a)-(c) show the time evolution of the strain-stress relations for three cases: (a) The $\mathscr{E}_{n 1}-P_{11}^{\mathrm{m}}$ relation obtained from the upper layer of the cross-sectional discretization. (b) Idem as in (a) but obtained from the bottom cross-sectional layer. (c) The (shear) strain-stress relation $\mathscr{E}_{n 3}-P_{13}^{\mathrm{m}}$ obtained from the layer located immediately over the geometrical center of the cross section. From these figures, it is clear that the obtained strain-stress relations strongly depend on the size of elements (as expected), but that the number of layers of the cross-sectional discretization has a minor significance.

Therefore, it is possible to affirm that regularizing the strain-stress relation according to the specific fracture energy and the characteristic length of the fractured zone (characteristic mesh size), the purpose of obtaining a mesh-independent response at global level is obtained, even when the post-yielding strain-stress relations become mesh dependent. 

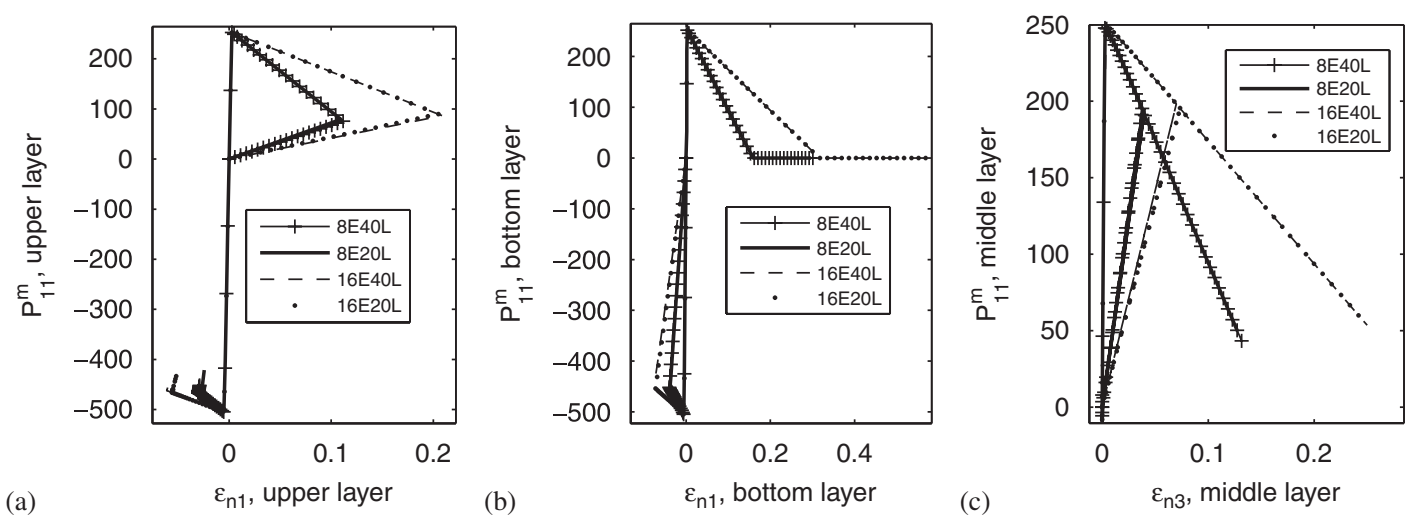

Figure 10. (a) Evolution of the $\mathscr{E}_{n 1}-P_{11}^{\mathrm{m}}$ relation in the upper layer of the cross section; (b) evolution of the $\mathscr{E}_{n 1}-P_{11}^{\mathrm{m}}$ relation in the bottom layer of the cross section; and (c) evolution of the $\mathscr{E}_{n 3}-P_{13}^{\mathrm{m}}$ relation in the layer of the middle of the cross section.

\subsection{Experimental-numerical comparative study of a scaled $R C$ building model}

This example corresponds to the comparison between the numerical simulation obtained by means of the proposed formulation and the experimental data obtained by $\mathrm{Lu} \mathrm{[68]} \mathrm{for} \mathrm{the} \mathrm{seismic} \mathrm{analysis}$ of a scaled model (1:5.5) of a regular bare frame. The structure was designed for a ductility class medium in accordance with the Eurocode 8 [69] with a peak ground acceleration of $0.3 g$ and soil profile A.

The material properties are: (i) Concrete class C20 with an elastic modulus of $E_{\mathrm{c}}=2 \times$ $10^{4} \mathrm{Nmm}^{-2}$, Poisson coefficient $v_{\mathrm{c}}=0.2$, characteristic compressive strength $f_{\mathrm{c}}^{-}=20 \mathrm{Nmm}^{-2}$ $\left(f_{\mathrm{c}}^{+}=2 \mathrm{Nmm}^{-2}\right.$ and $\left.K=1\right)$, fracture energy $G_{\mathrm{c}}^{-}=10 \mathrm{Nmm}^{-2}\left(G_{\mathrm{c}}^{+}=1 \mathrm{Nmm}^{-2}\right)$, mass density $\rho_{\mathrm{c}}=2.4 \times 10^{-9} \mathrm{Kg} \mathrm{mm}^{-3}$ and fluidity $\mu^{+}=1500 \mathrm{~s}^{-1}, \mu^{-}=5 \times 10^{4} \mathrm{~s}^{-1}\left(a^{ \pm}=1\right)$ and (ii) Flexural reinforcement Grade S400, $E_{\mathrm{S}}=2.1 \times 10^{5} \mathrm{Nmm}^{-2}, v_{\mathrm{s}}=0.3, f_{\mathrm{s}}^{ \pm}=400 \mathrm{Nmm}^{-2}(K=1), G_{\mathrm{s}}^{ \pm}=$ $100 \mathrm{Nmm}^{-2}, \rho_{\mathrm{s}}=7.8 \times 10^{-9} \mathrm{Kg} \mathrm{mm}^{-3}$ and $\mu^{ \pm}=5000 \mathrm{~s}^{-1}\left(a^{ \pm}=1\right)$. Other details such as loads, geometry and distribution of steel reinforcements can be consulted in the same publication. In the experimental program, the structure was subjected to several scaled versions of the $\mathrm{N}-\mathrm{S}$ component of the El Centro 1940 earthquake record.

Four quadratic elements with two Gauss integration points were used for each beam and column. Cross sections where meshed into a grid of 20 equally spaced layers. Longitudinal steel reinforcements were included in the external layers as part of a composite material. The fracture energy of the damage model used for concrete was modified to take into account the confining effect of transversal stirrups [16]. In the numerical simulations, the model is subjected to a pushover analysis. Static forces derived from the inertial contribution of the masses are applied at the floor levels considering an inverted triangular distribution.

A relationship between the measured base shear and the top lateral displacement is given in Reference [68] for each seismic record. This curve is compared in Figure 11 with the capacity curve obtained by means of using the numerical pushover analysis. Additionally, the result obtained from a numerical simulation presented by the authors in [70] using an alternative damage model is given as well. 


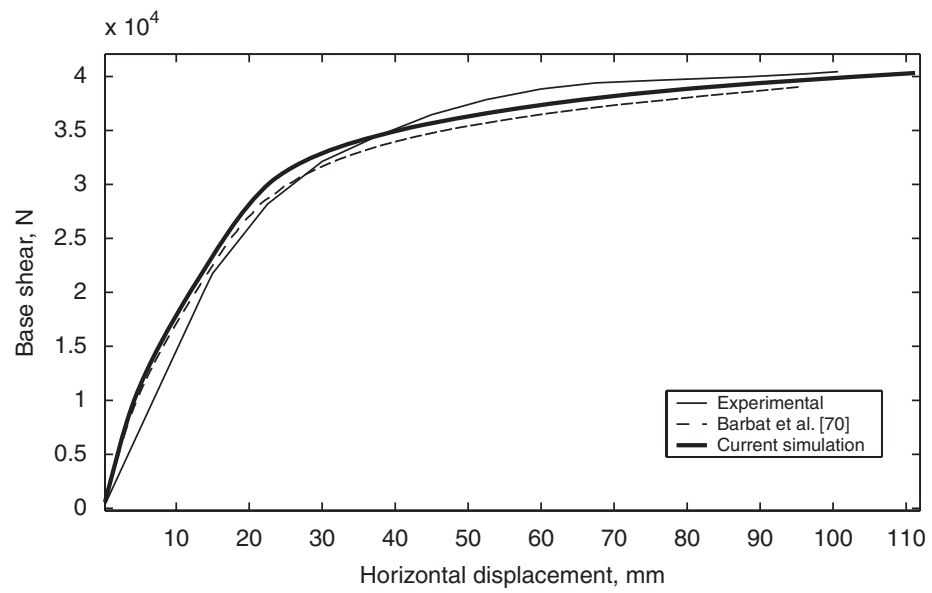

Figure 11. Capacity curves. Comparison between the results obtained from a numerical pushover analysis and experimental data.

It is possible to see that the pushover analysis gives a good approximation for the global maximum response and, therefore, it constitutes a suitable procedure for computing the non-linear behavior of structures subjected to seismic actions. In the same figure, it is possible to appreciate that in both the numerical simulation and the experimental data the characteristic values of the structure; that is, global ductility level, elastic limit and over-strength are similar.

Figure 12 shows a comparison between the distribution of cross-sectional damage predicted numerically and the map of cracking obtained after the application of several shaking table tests. The proposed damage index is able to reproduce the general failure mechanism of the structure where dissipation is mainly concentrated in the beam elements.

\subsection{Seismic response of a precast RC building with EDDs}

The non-linear seismic response of a typical precast RC industrial building shown in Figure 13 is studied. The building has a bay width of $24 \mathrm{~m}$ and $12 \mathrm{~m}$ of inter-axes length. The storey height is $10 \mathrm{~m}$. The concrete of the structure is H-35 with an elastic modulus of $E_{\mathrm{c}}=2.9 \times 10^{4} \mathrm{Nmm}^{-2}, v_{\mathrm{c}}=$ 0.2 , characteristic compressive strength $f_{\mathrm{c}}^{-}=35 \mathrm{~N} \mathrm{~mm}^{-2}\left(f_{\mathrm{c}}^{+}=3.5 \mathrm{Nmm}^{-2}\right.$ and $\left.K=1\right)$, fracture energy density $G_{\mathrm{c}}^{-}=20 \mathrm{Nmm}^{-2}\left(G_{\mathrm{c}}^{+}=5 \mathrm{Nmm}^{-2}\right)$, mass density $\rho_{\mathrm{c}}=2.4 \times 10^{-9} \mathrm{Kg} \mathrm{mm}^{-3}$ and fluidity $\mu_{\mathrm{c}}^{+}=1500 \mathrm{~s}^{-1}, \mu_{\mathrm{c}}^{-}=5 \times 10^{4} \mathrm{~s}^{-1}\left(a_{\mathrm{c}}^{ \pm}=1\right)$. In the case of the steel reinforcements, $E_{\mathrm{s}}=2.0 \times$ $10^{5} \mathrm{Nmm}^{-2}, v_{\mathrm{s}}=0.15, f_{\mathrm{s}}^{ \pm}=510 \mathrm{Nmm}^{-2}(K=1), G_{\mathrm{s}}^{ \pm}=500 \mathrm{Nmm}^{-2}, \rho_{\mathrm{s}}=7.8 \times 10^{-9} \mathrm{Kg} \mathrm{mm}^{-3}$ and $\mu_{\mathrm{s}}^{ \pm}=\infty\left(a_{\mathrm{s}}^{ \pm}=1\right)$. This figure also shows some details of the steel reinforcement of the cross sections. The dimensions of the columns are $60 \times 60 \mathrm{~cm}^{2}$. The beam has an initial height of $50 \mathrm{~cm}$ on the supports and $140 \mathrm{~cm}$ in the middle of the span. The permanent loads considered are $1000 \mathrm{Nm}^{-2}$ and the weight of upper half of the closing walls with $225.000 \mathrm{~N}$. The input acceleration is the same as in Example 6.2.

The building is meshed using eight quadratic elements with two Gauss integration points for each beam and column. The cross-sectional grid of fibers is shown in Figure 14. One integration point is used for each quadrilateral. The EDD was simulated by means of the model of Section 3.5 reproducing a purely plastic dissipative mechanism. The properties of the device were designed for 
(a)

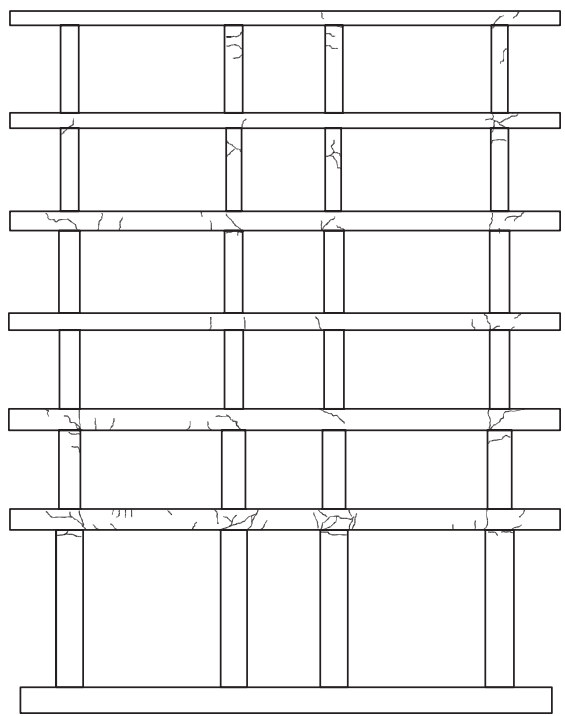

(b)

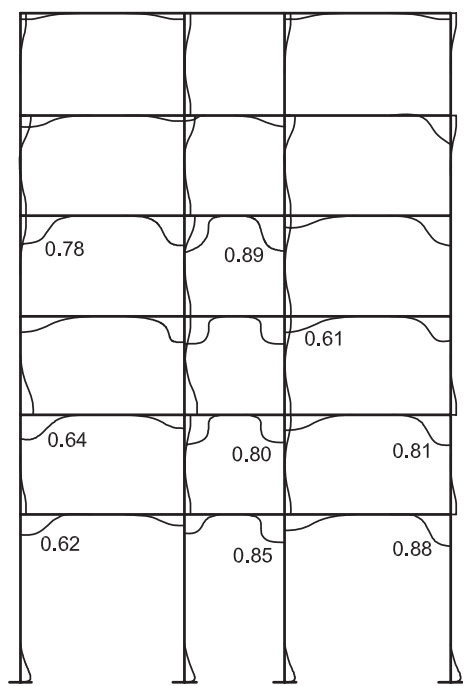

Figure 12. Damage: (a) map of fissures obtained from experimental data and (b) numerical: cross-sectional damage index.

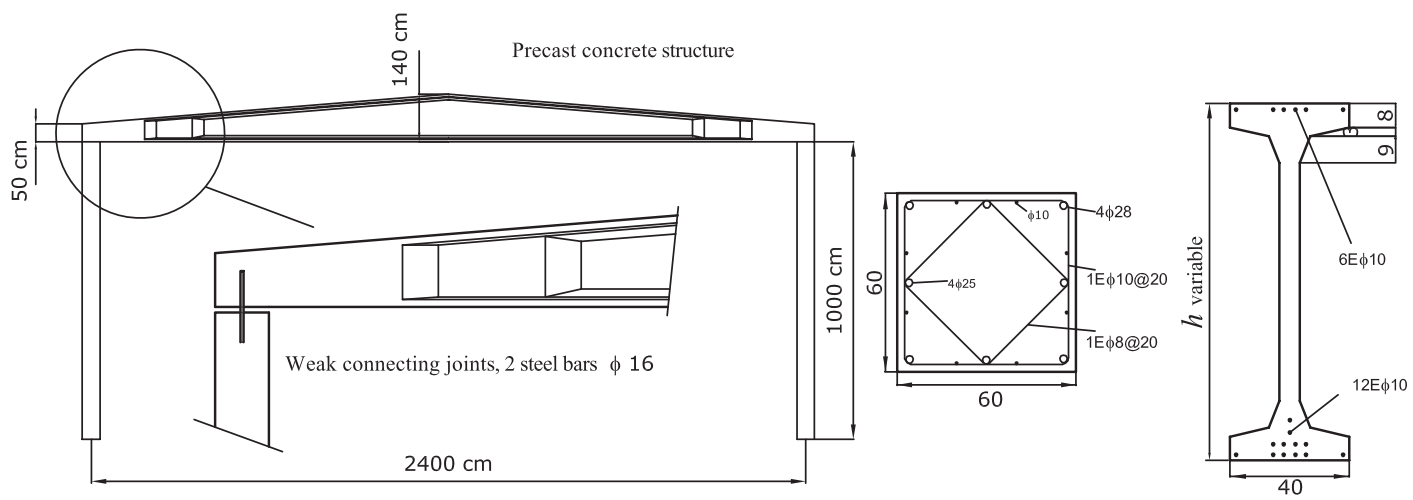

Figure 13. Description of the structure.

obtaining a yielding force of $200000 \mathrm{~N}$ for a relative displacement between the two end nodes of $1.2 \mathrm{~mm}$. Hardening or viscous effects were not considered. The length of the devices was of $3.1 \mathrm{~m}$.

First, a set of pushover analyses is performed considering the following cases: (i) The bare frame under small displacements assumption; (ii) The bare frame in finite deformation; (iii) The frame with EDDs and small deformation; (iv) Idem as (iii) but with finite deformation. The purpose is to establish clearly the importance of considering second-order effect coupled with inelasticity in the study of flexible structures.

Figure 15(a) shows the capacity curves obtained for the four mentioned cases. In this figure it is possible to see that for both, the passively controlled and uncontrolled cases, the small strain 


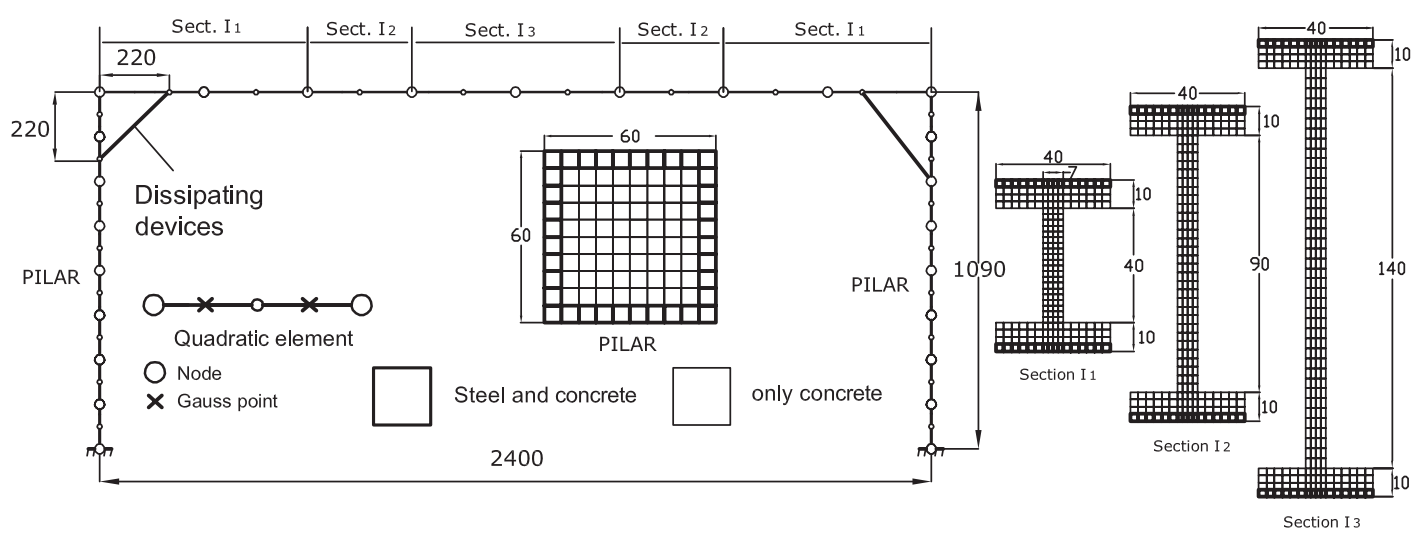

Figure 14. Numerical model of the precast frame with energy EDDs and cross-sectional meshes.
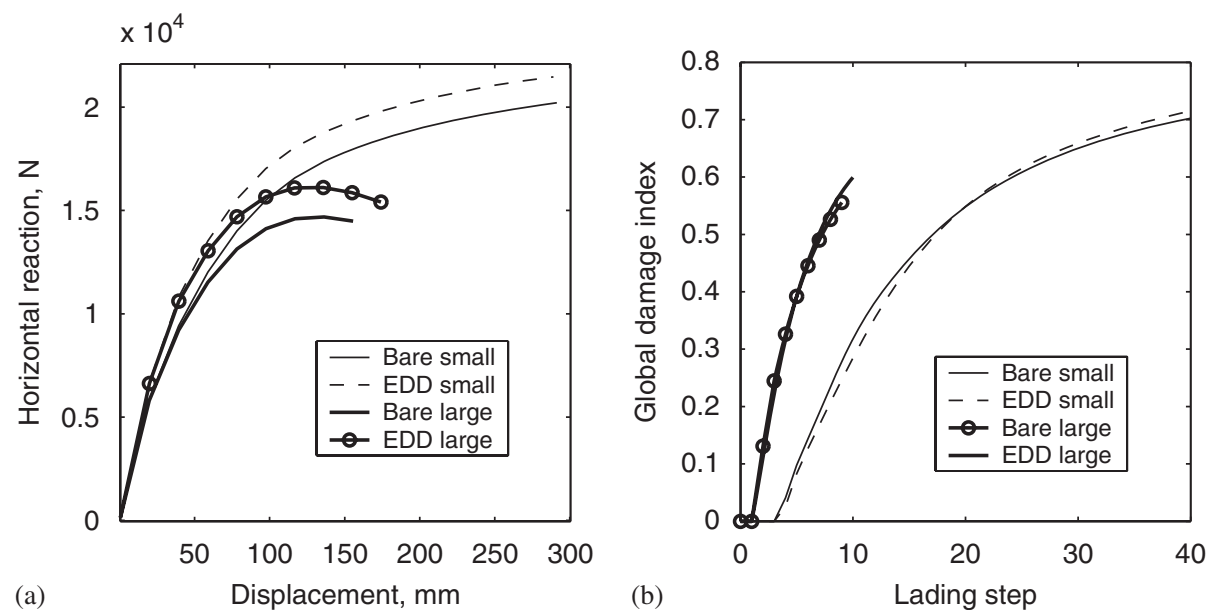

Figure 15. (a) Capacity curves and (b) evolution of the global damage index.

assumption overestimates the real load-carrying capacity of the structure, due to the fact that the vertical load derived from the self-weight compresses the columns, contributing to control the cracking and degradation due to the lateral loading. In the case of finite deformation, second-order effects are taken into account, the so-called $P-\Delta$ [71], and an anticipated strength degradation is observed for displacements over $60 \mathrm{~mm}$, which is a lateral displacement level expectable under strong seismic actions. Additionally, the incorporation of EDDs increases the stiffness and the yielding point of the structure at global level without affecting the global ductility. Although at material point level, softening is always present for the damage model beyond the linear-elastic limit, at global level only the simulation corresponding to case (iv) captures a small part of the softening post peak response.

Figure 15(b) shows the evolution of the global damage index for the cases (i)-(iv). Here it is possible to appreciate that the global damage index grows quickly for the cases when finite 


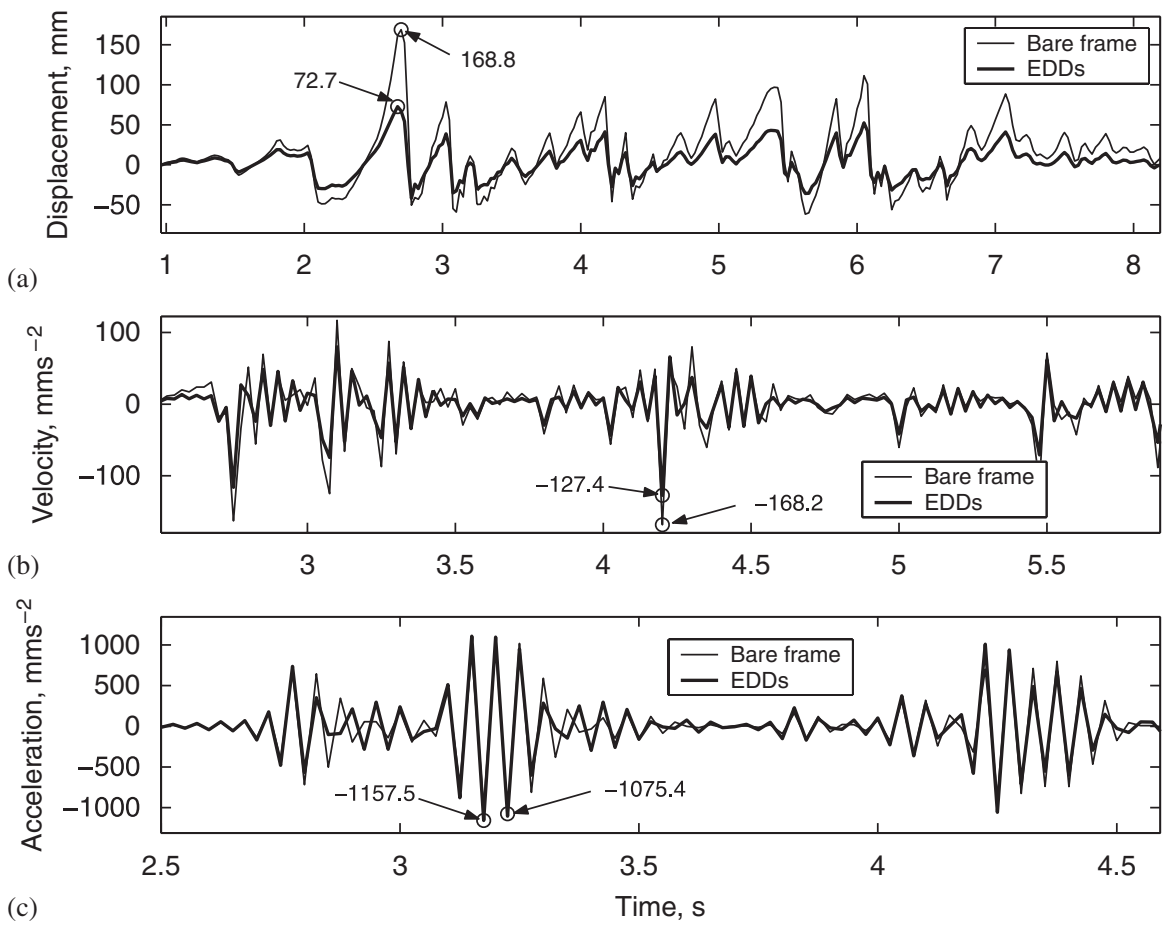

Figure 16. Time history responses of the top beam-column joint: (a) horizontal displacement; (b) velocity; and (c) acceleration.

deformation is considered and the benefits of adding EDDs are not visible due to the fact that the pushover analysis does not takes into account the effects of energy dissipation.

The results of the numerical simulations in the dynamic range allow seeing that the use of plastic EDDs contributes to improve the seismic behavior of the structure for the case of the employed acceleration record. Figure 16 shows the time history response of the horizontal displacement, velocity and acceleration of the upper beam-column joint for the uncontrolled and the controlled cases. A reduction of approximately $57.5 \%$ is obtained for the maximum lateral displacement when compared with the bare frame. Acceleration and velocity are controlled in the same way, but only 24.3 and $7.0 \%$ of reduction is obtained, respectively. A possible explanation for the limited effectiveness of the EDD is that the devices only contribute to increase the ductility of the beam-column joint without alleviating the base shear demand due to the dimensions of the device and its location in the structure.

Figure 17 shows the evolution of the global damage index. It is possible to see that the damage index grows quickly and reaching higher values for the non-controlled case, confirming the benefits obtained from the use of EDDs.

It is worth noting that with the present formulation the need of using specific cross-sectional constitutive laws depending on the cross-sectional shape and the distribution of steel reinforcements is completely avoided (compare with $[17,19]$ ). On the contrary, the constitutive relations are deduced using the cross-sectional integration procedure described in Section 5. Moreover, compared with other fiber models, [20], the coupling between stress components of thermodynamically 


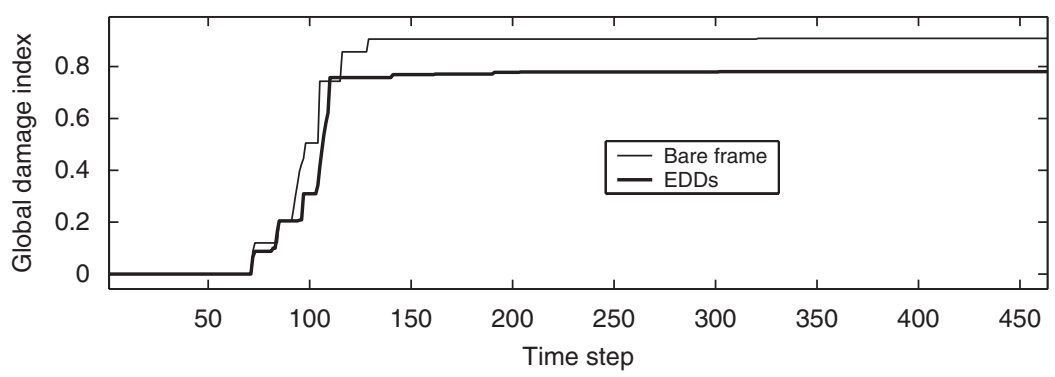

Figure 17. Evolution of the global damage index.

consistent constitutive laws allows to compute very precisely the dissipated energy and determine some damage variables.

\subsection{Seismic response of a three-dimensional urban building with EDDs}

This example corresponds to the study of the seismic non-linear response a 3-storey urban building designed according to the Eurocode 8 [69] for a ductility class medium, soil profile A and a peak ground acceleration of $0.2 \mathrm{~g}$. A three-dimensional view of the building is shown in Figure 18(e).

The main purpose of this example is to show the ability of the proposed formulation for simulating the geometric and constitutive non-linear behavior of a three-dimensional structure with and without EDDs, highlighting some complex phenomena such as the torsional response coupled with $P-\Delta$ effects for the case of a non-uniform distribution of mass.

The building is subjected to a scaled version of the $\mathrm{N}-\mathrm{S}$ component of the El Centro earthquake record corresponding to a peak ground acceleration of $0.3 g$, greater than the maximum horizontal load permitted by the design code. The record is applied in the $X$ direction (see Figure 18(c)). The mechanical properties for the steel and the concrete are the same as in Example 6.3. The details of the steel reinforcement are shown in Figure 18(d) according to the floor level where the beams and columns are located. Each beam and column is meshed using five equally spaced quadratic elements evaluated according to the Gauss integration rule and each cross section is divided into a $8 \times 8$ equally spaced grid of quadrilaterals.

Inertial forces derived from the contribution of the mass corresponding to a concrete floor of $130 \mathrm{~mm}$ thickness along with the sum of the dead and live loads of $2500 \mathrm{Nm}^{-2}$ (uniformly distributed on the floors) are considered. Structural torsion is induced adding two point masses, with values corresponding to the $10 \%$ of the total floor's mass, on the top corners $A$ and $B$ indicated in Figures 18(a)-(c). Therefore, even when the seismic action is applied in one direction, coupled displacement will appear in the another one due to the inhomogeneous distribution of masses.

Several practical aspects of the performance of an engineering structure are considered: structural torsion, distribution of the seismic damage, over-strength and ductility. Three cases are considered:

(i) Bare building (full geometric and constitutive non-linear model).

(ii) Elastic bare building equipped with viscous EDDs located in the $A-A$ and $C-C$ frames as indicated in Figures 18(a)-(c). Their mechanical properties are: (i) A linear viscous coefficient of $c_{d}=10000 \mathrm{~N} \mathrm{~s}^{-1}$ with (ii) an exponent $n=0.5$ (see Equations (45c) and (46)).

(iii) Full non-linear model of the building equipped with viscous EDDs (idem mechanical properties as in (ii)). 


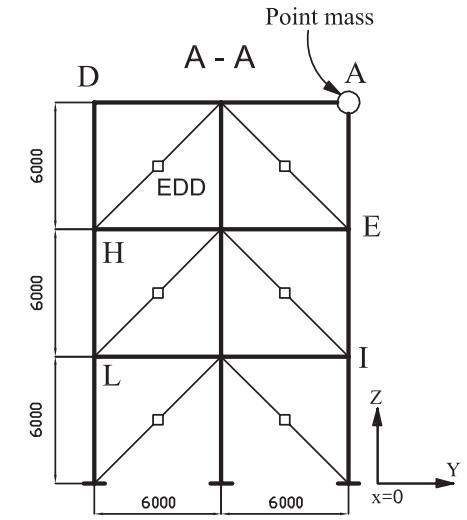

(a)

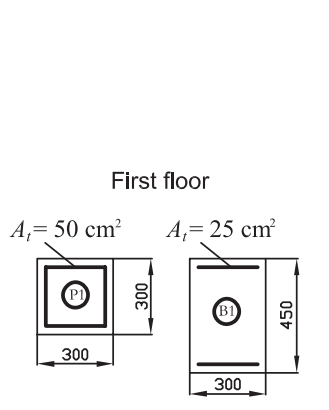

(d)

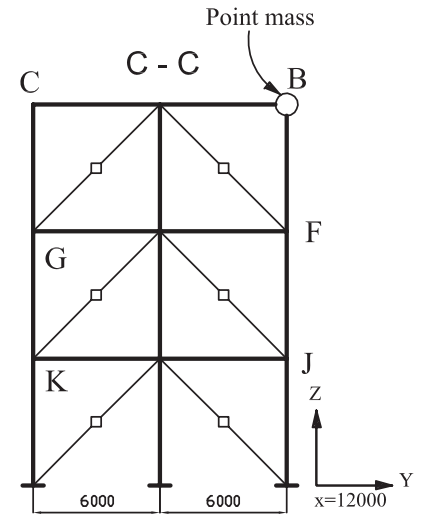

(b)

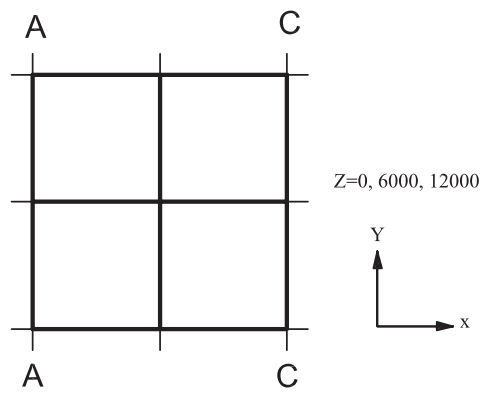

(c)

Figure 18. 3-storey urban building: (a) A-A plane frame showing the location of the EDDs; (b) C-C plane frame; (c) horizontal view showing the location of the A-A and $\mathrm{C}-\mathrm{C}$ frames; (d) RC detailing. $P 1$ and $B 1$ designate the steel reinforcement types of the columns and beams of the first floor $(P 2, B 2, P 3$ and $B 3$ idem as before but for the second and third floors, respectively); and (e) three-dimensional view.

The purpose of including the case (ii) is to study the influence on the structural response of the widely used (a priori) assumption that considers that the structure remains elastic as a consequence of the benefits obtained from EDDs.

The displacement time history responses of the nodes $B$ and $C$ of the building in the direction of the applied record are shown in Figure 19(a) and (b), respectively. The differences observed in these figures are due to the global torsion of the building. Additionally, it is possible to appreciate that the use of the proposed viscous dampers contributes to alleviate the maximum global displacement, decreasing the response by about $25 \%$. However, another important result is given by the fact that the a priori assumption that the main structure remains elastic underestimates the maximum displacement and, therefore, the ductility demand of the structural elements.

Figures 20(a) and (b) show the displacement time history response of the nodes $C$ and $D$ in the direction perpendicular to the application of the loading. Therefore, they are produced due to the torsion. The main two aspects that have to be highlighted in this case are: (i) Again the assumption of a linear bare structure is not able to capture appropriately the torsional components of the motion and (ii) the inclusion of viscous EDDs can alleviate significatively the torsional 

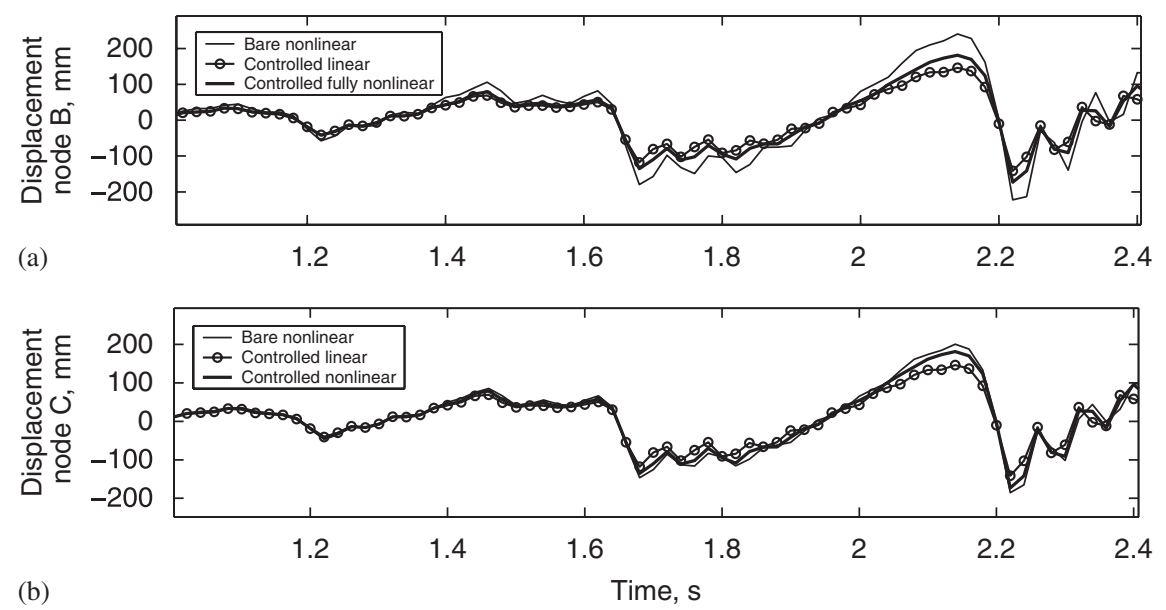

Figure 19. Displacement time history response in the direction of the applied record: (a) node $B$ and (b) node $C$.
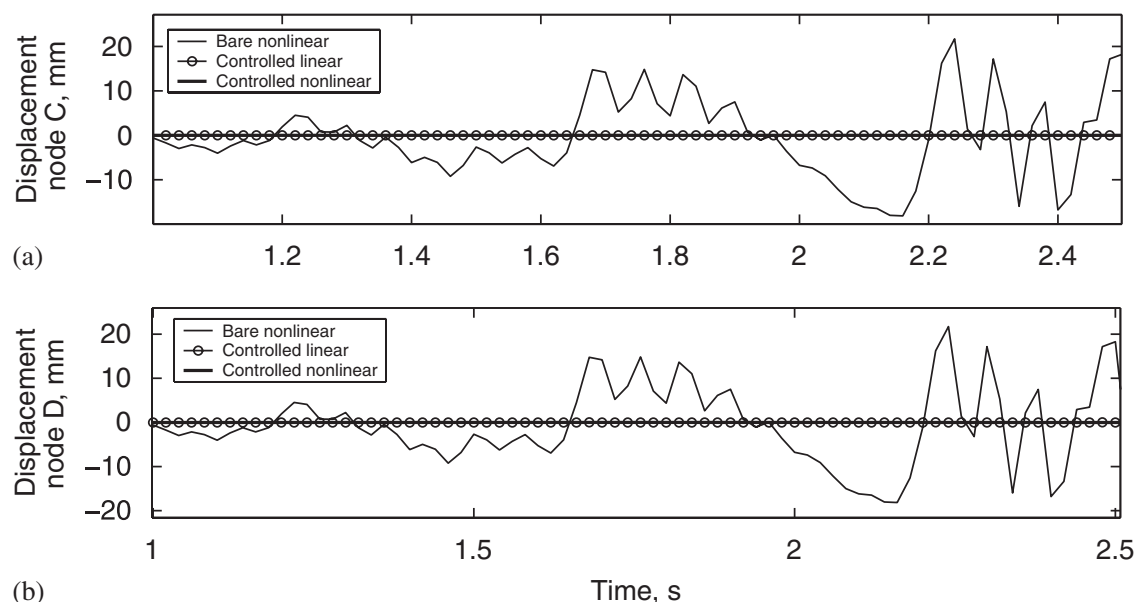

Figure 20. Displacement time history response in the direction of the applied record: (a) node $C$ and (b) node $D$.

response in the non-linear range. It has to be noted, however, that the masses added in the present example are rather small compared with the total one.

It is worth noting that damage appears in columns due to the poor seismic design considered (weak column-strong beams). The evolution of the global damage index is shown in Figure 21 for the full non-linear controlled and uncontrolled cases. In the same manner as before, the benefits obtained from the application of EDDs are clearly evidenced. 


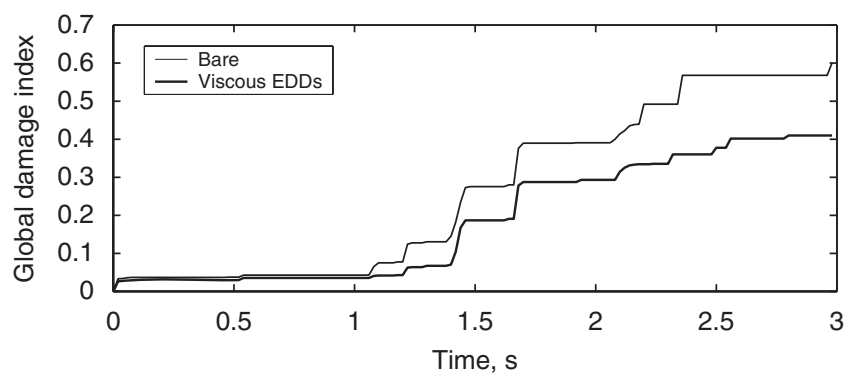

Figure 21. Time history response of the global damage index.

\section{CONCLUSIONS}

In this work, a geometrically exact formulation for initially curved and twisted beams has been extended for considering arbitrary distributions of rate-dependent inelastic composite materials on the cross sections in the static and dynamic (even seismic) cases. The consistent linearization of the weak form of the momentum balance equations considers the constitutive non-linearity with rate-dependent effects. The resulting model is implemented in a displacement-based finite element code. An iterative Newton-Raphson scheme is used for the solution of the time-discrete version of the linearized problem. A specific finite element for EDDs is developed based on the beam model, but releasing the rotational degrees of freedom.

Each material point of a cross section is assumed to be composed of several simple materials with their own constitutive laws. Thermodynamically consistent versions of the plasticity and the tensile-compressive damage models are developed, which consider full interaction among the components of the FPK stress vector. The mixing rule is used for describing the resulting composite. Viscosity is included at constitutive level by means of an adequate regularization of the evolution equations of the damage thresholds, avoiding ad hoc formulations such as the so-called Rayleigh's method (see [72]). A versatile model is proposed for the force-displacement relation of the EDDs. Calibrating the model's parameters it is possible to simulate a wide variety of dissipative mechanisms.

From the numerical point of view, beam cross sections are meshed into a grid of quadrilaterals corresponding to fibers directed along the beam axis. Then, in each integration point, two additional integration loops are required to obtain the reduced cross-sectional forces, moments and tangential tensors. The present approach avoids the formulation of constitutive laws in terms of cross-sectional quantities, which in most of the cases are valid for specific geometries and the thermodynamic basis of the constitutive laws are, in general, violated. Moreover, the shear components of the stress are determined from the inelastic constitutive laws and, therefore, they are not deduced from the elemental equilibrium as in the case of other fiber models [20]. Local and global damage indices have been developed based on the ratio between the non-linear stresses and their visco elastic counterparts. They constitute a measure of the degradation level and, therefore, of the dissipated energy.

The present formulation is validated by means of a set of numerical examples, which includes the comparison with existing experimental data and the study of the seismic response of precast and cast in place RC structures equipped with EDDs. Several rather complex phenomenon can 
be reproduced: (i) The coupling between geometric and constitutive non-linearity ( $P-\Delta$ effects) in the static and dynamic ranges; (ii) The full non-linear dynamic torsional response for structures presenting non-coincident elasticity and mass centers subjected to seismic loadings; (iii) The numerical simulation and evaluation (in terms of the evolution of damage indices) of the effectiveness of using passive EDDs for improving the dynamic response of RC buildings.

The use of EDDs appears as a convenient technique for controlling the dynamic behavior of RC structures subjected to earthquake loading; however, for strong seismic actions an appreciable level of damage can also be produced in the main structural elements; therefore, this aspect should be considered when designing passively controlled structures.

\section{ACKNOWLEDGEMENTS}

This research was partially supported by the European Commission, CEE-FP6 Project FP6-50544(GOCE) 'Risk Mitigation for Earthquakes and Landslides (LESSLOSS)'; by the Spanish Government (Ministerio de Educación y Ciencia), project BIA2003-08700-C03-02 'Numerical simulation of the seismic behavior of structures with energy dissipation devices', project BIA2005-06952 'Study of composite materials for design, reinforcement and retrofit of civil engineering structures (RECOMP)'; and by the collaboration framework between CIMNE and AIRBUS, project PBSO-13-06 'Innovative finite element methods for non-linear analysis of composite structures (FEMCOM)'. All this support is gratefully acknowledged.

\section{REFERENCES}

1. Hanson RD, Aiken ID, Nims DK, Ritchter PJ, Batchman RE. State of the art and state of the practice in seismic engineering dissipation. Proceedings, ATC-17-1 Seminar on Seismic Isolation, Passive Energy Dissipation and Active Control, Applied Technology Council, San Francisco, CA, March 1993.

2. Pampanin S, Calvi GM, Moratti M. Seismic behavior of RC beam-column joints designed for gravity only. Twelfth European Conference on Earthquake Engineering, London, 9-13 September 2002. Paper Reference 726.

3. Reinhorn AM, Constantinou MC, Li C. Use of supplemental damping devices for seismic strengthening of lightly reinforced concrete frames. Proceedings, Workshop on the Seismic Rehabilitation of Lightly Reinforced Concrete Frames, Gaithersburg, MD, 12-13 June 1995.

4. Soong TT, Dargush GF. Passive Energy Dissipation Systems in Structural Engineering. Wiley: New York, 1997.

5. Fu Y, Kasai K. Comparative study of frames using viscoelastic and viscous dampers. Journal of Structural Engineering 1998; 124(5):513-522.

6. Kasai K, Fu Y, Watanabe A. Passive control systems for seismic damage mitigation. Journal of Structural Engineering 1998; 124(5):501-512.

7. Aiken I. Passive energy dissipation hardware and applications. Proceedings, Los Angeles County and Seaosc Symposium on Passive Energy Dissipation Systems for New and Existing Buildings, Los Angeles, July 1996.

8. Lin YY, Chang KC. Study on damping reduction factors for buildings under earthquake ground motions. Journal of Structural Engineering 2003; 129(2):206-214.

9. Connor JJ, Wada A, Iwata M, Huang YH. Damage-controlled structures. I: preliminary design methodology for seismically active regions. Journal of Structural Engineering 1997; 123(4):423-431.

10. Lin WH, Chopra AK. Asymmetric one-storey elastic system with non-linear viscous and viscoelastic dampers: earthquake response. Earthquake Engineering and Structural Dynamics 2003; 32:555-577.

11. Clark P, Aiken I, Ko E, Kasai K, Kimura I. Design procedures for buildings incorporating hysteretic seismic devices. Proceedings, 68th Annual Convention, Structural Engineering Association of California, Santa Barbara, CA, October 1999.

12. Federal Emergency Management Agency (FEMA). Prestandard and commentary for the seismic rehabilitation of buildings. Report 356, Washington, DC, FEMA, 2000.

13. Federal Emergency Management Agency (FEMA). NEHRP recommended provisions for seismic regulations for new buildings and other structures. Report 368, Washington, DC, FEMA, 2000. 
14. Shen KL, Soong TT. Design of energy dissipation devices based on concept of damage control. Journal of Structural Engineering 2005; 122(1):76-82.

15. Mata P, Barbat AH, Oller S, Boroschek R. Computational models for the seismic response of reinforced concrete buildings with energy dissipating devices. Archives of Computational Methods in Engineering 2008; 15:489-539. DOI: $10.1007 / \mathrm{s} 11831-008-9024-\mathrm{z}$.

16. Mata P, Oller S, Barbat AH. Static analysis of beam structures under nonlinear geometric and constitutive behavior. Computer Methods in Applied Mechanics and Engineering 2007; 196:4458-4478.

17. Bayrak O, Sheikh SA. Plastic hinge analysis. Journal of Structural Engineering 2001; 127(9):1092-1100.

18. Simo JC, Hjelmstad KD, Taylor RL. Numerical formulations of elasto-viscoplastic response of beams accounting for the effect of shear. Computer Methods in Applied Mechanics and Engineering 1984; 42:301-330.

19. Sivaselvan MV, Reinhorn AM. Collapse analysis: large inelastic deformations analysis of planar frames. Journal of Structural Engineering 2002; 128:1575-1583.

20. Nukala PKVV, White DW. A mixed finite element for three-dimensional nonlinear analysis of steel frames. Computer Methods in Applied Mechanics and Engineering 2004; 193(5):2507-2545.

21. Ibrahimbegović A. On finite element implementation of geometrically nonlinear Reissner's beam theory: threedimensional curved beam elements. Computer Methods in Applied Mechanics and Engineering 1995; 122:11-26.

22. Simo JC. A finite strain beam formulation. The three-dimensional dynamic problem. Part I. Computer Methods in Applied Mechanics and Engineering 1985; 49:55-70.

23. Mata P, Oller S, Barbat AH. Dynamic analysis of beam structures considering geometric and constitutive nonlinearity. Computer Methods in Applied Mechanics and Engineering 2008; 197:857-878.

24. Reissner E. On one-dimensional large-displacement finite-strain beam theory. Studies in Applied Mathematics 1973; LII 2:87-95.

25. Simo JC, Vu-Quoc L. On the dynamics in space of rods undergoing large motions-a geometrically exact approach. Computer Methods in Applied Mechanics and Engineering 1988; 66:125-161.

26. Mata P, Boroschek R, Barbat AH, Oller S. High damping rubber model for energy dissipating devices. Journal of Earthquake Engineering 2007; 11(2):231-256.

27. Simo JC, Vu-Quoc L. A three-dimensional finite-strain rod model. Part II: computational aspects. Computer Methods in Applied Mechanics and Engineering 1986; 58:79-116.

28. Kapania RK, Li J. On a geometrically exact curved/twisted beam theory under rigid cross-section assumption. Computational Mechanics 2003; 30:428-443.

29. Antman SS. Nonlinear Problems of Elasticity. Springer: Berlin, 1991.

30. Ortiz M, Popov E. Plain concrete as a composite material. Mechanics of Materials 1982; 1:139-150.

31. Car E, Oller S, Oñate E. An anisotropic elasto plastic constitutive model for large strain analysis of fiber reinforced composite materials. Computer Methods in Applied Mechanics and Engineering 2000; 185:245-277.

32. Oller S, Oñate E, Miquel J. Mixing anisotropic formulation for the analysis of composites. Communications in Numerical Methods in Engineering 1996; 12:471-482.

33. Bratina S, Saje M, Planinc I. On materially and geometrically non-linear analysis of reinforced concrete planar frames. International Journal of Solids and Structures 2004; 41:7181-7207.

34. Davenne L, Ragueneau F, Mazar J, Ibrahimbegović A. Efficient approaches to finite element analysis in earthquake engineering. Computers and Structures 2003; 81:1223-1239.

35. Kachanov L. Time of rupture process under creep conditions. Izvestia Akademii Nauk SSSR, Otdelenie Tekhnicheskich Nauk 1958; 8:26-31.

36. Chaboche J. Continuum damage mechanics. Part I: general concepts. Journal of Applied Mechanics (ASME) 1988; 55:59-64.

37. Chaboche J. Continuum damage mechanics. Part II: damage growth, crack initiation, and crack growth. Journal of Applied Mechanics (ASME) 1988; 55:65-72.

38. Krajcinovic D, Fonseka G. The continuum damage theory of brittle materials. Part 1: general theory. Journal of Applied Mechanics (ASME) 1981; 48:809-815.

39. Krajcinovic D. Damage mechanics: accomplishments, trends and needs. International Journal of Solids and Structures 2000; 37:267-277.

40. Pijaudier-Cabot G, Mazars J. Damage models for concrete. In Handbook of Materials Behavior, Chapter 6, Lemaitre J (ed.), vol. 2. Academic Press: New York, 2001; 500-512.

41. Mazars J, Pijaudier-Cabot G. Continuum damage theory. Application to concrete. Journal of Engineering Mechanics (ASCE) 1989; 115(2):345-365.

42. Simo JC. On a fully three-dimensional finite-strain viscoelastic damage model: formulation and computational aspects. Computer Methods in Applied Mechanics and Engineering 1987; 60:153-173. 
43. Lemaitre J. Coupled elasto-plasticity and damage constitutive equations. Computer Methods in Applied Mechanics and Engineering 1985; 51:31-49.

44. Menzel A, Ekh M, Steinmann P, Runesson K. Anisotropic damage coupled to plasticity: modelling based on the effective configuration concept. International Journal for Numerical Methods in Engineering 2002; 54:1409-1430.

45. Armero F, Oller S. A general framework for continuum damage models. I. Infinitesimal plastic damage models in stress space. International Journal of Solids and Structures 2000; 37:7409-7436.

46. De Souza Neto EA, Perić D, Owen DRJ. Continuum modelling and numerical simulation of material damage at finite strains. Archives of Computational Methods in Engineering 1998; 5(4):311-384.

47. Faria R, Oliver J, Cervera M. A strain-based plastic viscous-damage model for massive concrete structures. International Journal of Solids and Structures 1998; 35(14):1533-1558.

48. Lubliner J. On the thermodynamic foundations of non-linear solid mechanics. International Journal of Non-Linear Mechanics 1972; 7:237-254.

49. Lubliner J. Thermomechanics of Deformable Bodies. Department of Civil Engineering, University of California Press: Berkeley, 1985.

50. Simo JC, Ju JW. Strain- and stress-based continuum damage models-II. Computational aspects. International Journal of Solids and Structures 1987; 23(7):841-869.

51. Barbat AH, Oller S, Hanganu A, Oñate E. Viscous damage model for Timoshenko beam structures. International Journal for Solids and Structures 1997; 34(30):3953-3976.

52. Hanganu AD, Oñate E, Barbat AH. Finite element methodology for local/global damage evaluation in civil engineering structures. Computers and Structures 2002; 80:1667-1687.

53. Simo JC, Ju JW. Strain- and stress-based continuum damage models-I. Formulation. International Journal of Solids and Structures 1987; 23(7):821-840.

54. Cercera M. Viscoelasticity and rate-dependent continuum damage models. Pub. M79, International Center for Numerical Methods in Engineering, Barcelona, 2003.

55. Cervera M, Oliver J, Prato T. Thermo-chemo mechanical model of concrete. I: hydration and aging. Journal of Engineering Mechanics (ASCE) 1999; 125:1018-1027.

56. Cervera M, Oliver J, Manzoli O. A rate-dependent isotropic damage model for the seismic analysis of concrete dams. Earthquake Engineering and Structural Dynamics 1996; 25(9):987-1010.

57. Oliver J, Cervera M, Oller S, Lubliner J. Isotropic damage models and smeared crack analysis of concrete. Proceedings 2nd ICCAADCS, Zell Am See, vol. 2. Pineridge Press: Austria, 1990; 945-958.

58. Oliver J. A consistent characteristic length for smeared cracking models. International Journal for Numerical Methods in Engineering 1989; 28:461-474.

59. Perry SH, Al-Shaikh AH, Cheong HK. Influence of strain rate on laterally confined concrete columns subjected to cyclic loading. Journal of Materials Research 1986; 1(2):382-388.

60. Simo JC, Hughes TJR. Computational Inelasticity, vol. 7. Springer: Berlin, 1997.

61. Oller S, Oñate E, Miquel J, Botello S. A plastic damage constitutive model for composites materials. International Journal of Solids and Structures 1996; 33(17):2501-2518.

62. Truesdell C, Toupin R. The Classical Field Theories. Handbuch der Physik III/I. Springer: Berlin, 1960.

63. Car E. Modelo constitutivo continuo para el estudio del comportamiento mecánico de los materiales compuestos. Ph.D. Thesis, Technical University of Catalonia, 2000.

64. Armero F. Energy-dissipative momentum-conserving time-stepping algorithms for finite strain multiplicative plasticity. Computer Methods in Applied Mechanics and Engineering 2006; 195:4862-4889.

65. Noels L, Stainier L, Ponthot JP. A first-order energy-dissipative momentum-conserving scheme for elasto-plasticity using the variational updates formulation. Computer Methods in Applied Mechanics and Engineering 2008; 197:706-726.

66. Simo JC, Tarnow N, Doblare M. Non-linear dynamics of three-dimensional rods: exact energy and momentum conserving algorithms. International Journal for Numerical Methods in Engineering 1992; 34:117-164.

67. Romero I, Armero F. An objective finite element approximation of the kinematics and geometrically exact rod and its use in the formulation of an energy-momentum conserving scheme in dynamics. International Journal for Numerical Methods in Engineering 2002; 54:1683-1716.

68. Lu Y. Comparative study of seismic behavior of multistory reinforced concrete framed structures. Journal of Structural Engineering 2002; 128(2):169-178.

69. EC8, Eurocode No 8. Design of structures for earthquake resistance, 3rd Draft, prEN 1998-1-1:2001, European Committee for Standardization, May 2001. 
70. Barbat AH, Mata P, Oller S, Vielma JC. Computational simulation of the seismic response of buildings with energy dissipating devices. ECCOMAS, Thematic Conference on Computational Methods in Structural Dynamics and Earthquake Engineering, Rethymno, Crete, Greece, 13-16 June 2007.

71. Williamson EB. Evaluation of damage and $P-\Delta$ effects for systems under earthquake excitation. Journal of Structural Engineering 2003; 129(8):1036-1046.

72. Chopra AK. Dynamics of Structures: Theory and Applications to Earthquake Engineering. Prentice-Hall: Englewood Cliffs, NJ, 2000. 